九州大学学術情報リポジトリ

Kyushu University Institutional Repository

\title{
Fluctuation Effects in Coagulation Processes
}

\section{早川，尚男}

九州大学理学研究科物理学専攻

https://doi.org/10.11501/3054122

出版情報: 九州大学，1990，理学博士，課程博士 バージョン：

権利関係 : 


\section{Fluctuation Effects in Coagulation Processes}




\section{Thesis}

Fluctuation Effects in Coagulation Processes

Department of Physics, Kyushu University, Fukuoka 812

Hisao Hayakawa 


\begin{abstract}
We formulate a theory of coagulation and develop the diagrammatic perturbation technique based on the Fock space formalsim. For a system of finite volume fraction of droplets $\phi$, we find that the screening length plays an important role, where the screening length is proportional to $\phi^{-1 / 2}$ for a three dimensional system and has the logarithmic dependence on $\phi$ for a two dimensional system.

We calculate the effective coagulation rate for diffusion-controlled coagultion and find the followings: The coagulation rate has correction to Smoluchowski's classical result in a medium of three dimension. This correction term is given by the ratio of the reaction radius to the screening length. For a two dimensional system, the coagulation rate is determined by a self-consistent condition and there is a logarithmic correction in time.

We also calculate the scaling form for the scattering function and discuss its volume fraction dependence for a three dimensional system. We obtain qualitative agreements with experimental data of binary fluid mixture and previous phenomenological theories for the peak height, the full width of half maximum, and the moment ratio of the scaling function.
\end{abstract}




\section{Acknowledgment}

I would like to express my sincere gratitude to Prof.K.Kawasaki for his encouragement and suggestions during my study of physics. Stimulative discussion between us generates this thesis. I thank Prof.K.Sekimoto(Nagoya), Dr.T.Kawakatsu and other members in our research group in Kyushu University for their helpful discussions.

I thank Prof.F.Family (Emory) for his invitation to the United States and his collaborations. Discussion with Dr.J.G.Amar (Emory) was useful for me. I will not forget Prof.H.'Takayasu (Kobe) who intiated me into the theory of coagulation. I thank Profs. II.'Tomita (Kyoto), A.Onuki (YI'TP), H.Furukawa (Yamaguchi) and A.J.Bray (Manchester) for their useful comments. Stimulative discussions with Profs. M.Doi (Nagoya), 'T.Ohta (Ochanomizu), Y.Oono (Illinois), H.J.Herrmann (Saclay), H.M.Lindsay ( Emory ), L.P.Kadanoff (Chicago), and G.F.Mazenko ( Chicago ), Drs.P.Meakin (du Pont) and T.Vicsek (Emory), Mr.R.Boide (Emory), S.Sasa (Kyoto) and H.Kohno (Kyoto) are acknowledged.

I thank warm hospitality during my stay at Emory University (1989.1-7) and the Yukawa Institute for Theoretical Physics(1990.6-7) where part of my work was carried out. My work has been partially supported by the Office of Naval Research and the Petrolem Fund, administrated by the American Chemical Society, and the Fellowships of the Japan Society for the Promotion of Science for Japanese Junior Scientists.

I express my gratitude my wife and my parents for their help for my life. In particular, my father teachs me what physics is. He has also navigated me to the right direction to become a physist. I have learned physics very much from him. I would like to devote this thesis to him. 


\section{Contents}

Astract

Acknowledgment

Contents

Chapter I: General Introduction

$\S$ I-1. Introductoin: What is Coagulation?

§ I-2. Coagulation in Phase Separations 8

§ I-3. Scaling Concepts in Coagulation 13

$\S$ I-4. Modern 'Theory of Coagulation: Beyond Smoluchowski's Theory 15

$\begin{array}{ll}\text { References in Chapter I } & 19\end{array}$

Chapter II: Fluctuation Effects in Coagulation Processes 23

$\S$ II-1. Introduction 24

$\S$ II-2. Basic Formalism $\quad 27$

§ II-3. The Diagrammatic Perturbation $\quad 31$

$\S$ II-4. The Effective Reaction Rate 34

$\S$ II-5. Scaling in the Scattering Function 40

§ II-6. Concluding Remarks $\quad 45$

$\begin{array}{ll}\text { References in Chapter II } & 48\end{array}$

$\begin{array}{ll}\text { Figures and Figure Captions in Chapter II } & 51\end{array}$

Appendix A: Classical Theory of Coagulation Processes 62

$\S$ A-1. Diffusion-Controlled Coagulation $\quad 62$

$\S$ A-2. Shear-Induced Coagulation 65

$\S$ A-3. The Effect of Force Field to Brownian Coagulation 66 
Appendix B: Exact Solutions of Smoluchowski's Rate Equation

$\S$ B-1. Exact Solution for $K_{i j}=1$

$\S$ B-2. Exact Solution for $K_{i j}=i j$ 71

$\S$ B-3. Exact Solution for $K_{i j}=i+j$ 74

References in Appendices 76

Figures and Figure Captions in Appendices 77 


\section{Chapter I: General Introduction}

\section{$\S$ I-1. Introduction: What is Coagulation?}

Coagulation is an irreversible physical process in which initially dispersed basic units (monomers) stick together to form clusters [1]. 'This process is also called aggregation or flocculation in some texts. 'Two fundamental aspects retain our attention. 'The first is the geometrical aspect, that is, the quantitative description of the structure of aggregates. 'The second is the kinetic aspect, that is, the quantitative description of the time evolution of statistical quantities such as the mean cluster size, size distribution and correlation functions. Recently, the first aspect has been the subject of a great amount of interest. These recent progresses are essentially due to the new mathematical concept, fractal [2], which permits the quantitative description of aggregates (aggregated clusters). On the other hand, the second aspect is also important and has long history since Smoluchowski $[3,4]$ proposed the kinetic rate equation in 1916.

Coagulation occurs quite commonly in many fields of science and technology. For example, such behavior can be observed for dust formation in air [5] or in acqeous gold

colloids [6]. In addition, examples are found in polymerization [7], the growth of interstellar dust grain in astrophysics[8], red blood cell coagulation in biology [9] and phase separations in metallurgy[10] or chemical physics $[11,12]$. Recently, a rapid growth of interest has taken place since the progress of computational simulation of colloidal aggregates [13]. Consequently, this process is called cluster-cluster aggregation (CCA). A typical computer simulation is started from randomly distributed monomers. At each time step a cluster moves by one lattice unit in a randomly chosen direction. Two clusters stick together 
to form a larger cluster. This simple process leads to fractal aggregates similar to that observed by experiments in gold colloids. The description of such a process is fascinating. However, theoretical description for fractal aggregates is very difficult compared to spherical clusters (droplets).

In kinetic description coagulation is represented as follows

$$
X_{i}+X_{j} \rightarrow X_{i+j}
$$

where a cluster containing $i$ monomers $(i-m e r)$ denotes $X_{i}$. Actually, as a description of a general coagulation process, (1.1.1) is a simplification. In practice the reverse reaction $X_{i+j} \rightarrow X_{i}+X_{j}$ occurs. Fragmentation can be taken into account $[14,15]$, but for our purposes this would lead to needless complications. For this reason we restrict ourselves to irreversible coagulation. In many cases the force between clusters is short ranged and it is strong enough to bind small clusters irreversibly when they contact each other. Even in the existence of the Coulomb force the Debye screening ensures that the effective force is short ranged and its effect is not so large, at least, for weakly charged cases. In the classical theory, correction due to force among clusters appears in a multiplied constant (see $A$ ppendix A). Therefore, we restrict ourselves to interaction among clusters of a hard core type in the proceeding discussions.

'The usual description of kinetic aspect in coagulation is based on Smoluchowski's rate equation. Introducing the rate constant for coagulation $K_{i j}$ the concentration of $s$-mer, $c_{s}(t)$, satisfies the following set of coupled rate equations

$$
\frac{\partial c_{s}(t)}{\partial t}=\frac{1}{2} \sum_{i+j=s} K_{i j} c_{i}(t) c_{j}(t)-\sum_{j=1}^{\infty} K_{s j} c_{s}(t) c_{j}(t),
$$

where $K_{i j}$ depends on physical processes. Equation (I.1.2) is known as Smoluchowski's rate equation. It has a simple interpretation. The first term (gain term) in the right hand 
side of (I.1.2) states that $s$-mer is formed out of possible combinations of $i$ - and $j$-mers which satisfy $i+j=s$. Similarly the second term (loss term) describes the loss of $s$-mer due to reactions of $s-$ mer with other clusters. The rate equation, of course, contains only information for size of clusters, while it does not contain spatial correlations and cluster's shape. If we involve information for cluster's shape, we cannot use a simple description like (I.1.2), because clusters with the same size have different shapes and the summation of their sizes become meaningless. 'Therefore we should discard an ambition to describe theoretically both geometry and kinetics.

In some special cases clusters have spherical forms due to the existence of surface tension. In such cases the system is in local equilibrium at the cluster's surface. Therefore, cluster's shape is relaxed to a spherical form to minimize the surface energy. We call such clusters droplets. Droplets can be observed in the course of phase separation of binary fluid mixtures $[11,12]$. We mainly focus on coagulation process of droplets for simplification of our discussions.

The arrangement of this thesis is as follows. First, we explain the contents in this chapter. In section I-2, we briefly explain the role of coagulation in phase separation. In section I-3 we introduce a key concept in coagulation process, namely, dynamical scaling law. We explain the outline of scaling properties in coagulation focusing on common aspects which are independent of a particular process. In section I-4 we explain the motivation of our work and give a briel survey of extension of the classical theory. This section can be regarded as an introduction to the next chapter which contains our original work. In the course of chapter I it becomes clear that the papers previously published by the present author have been written under a unified point of views. 
We show the contents in the next chapter. The chapter II is the central part of this thesis which is also submitted to Physica A [12]. Section II-1 is the introduction of this chapter II. The classical theory by Smoluchowski[3] is explained briefly and we indicate crucial points of his argument. In section II-2 we formulate a theory of coagulation using the Fock space formalism [16]. In section II-3 we develop the diagrammatic technique to calculate statistical quantities. The essential part of the above sections can be found in the previous paper [17]. In section II-4 we obtain the effective coagulation rate and show that there is a correction term to Smoluchowski's classical theory even for $d=3$. We also show the existence of the logarithmic correction in two dimensional coagulation systems. In section II-5 we calculate the scaling form for the scattering function and compare our results with experimental data for binary fluid mixtures[18]. In section II-6 we present the concluding remarks on this chapter.

In Appendices we present supplementary explanations to the text [4,5]. In Appendix $\Lambda$ we derive coagulation rate constants for diffusion-controlled case and shear-induced case within the framework of classical theory. We also derive a correction term to the classical theory using an effective medium theory. In addition, we discuss the effects of interaction among droplets. In Appendix B, we illustrate exact solutions of Smoluchowski's rate equation (I.1.2), where reaction kernels are $K_{i j}=1[3,4], i+j[19]$ and $i j[7]$ in a certain unit.

\section{$\S$ I-2. Coagulation in Plıase Separations}

In this section, we give a brief review of phase separation. We clarify the role of coagulation in phase separations. 
Phase separation is the ordering process undergoing a first-order phase transition [20]. In a typical situation, a system is rapidly quenched from a one-phase equilibrium state to a nonequilibrium state inside the coexistence curve. Such a quenched system gradually evolves from the nonequilibrium state to an equilibrium state consisting of two coexisting phases. Phase separation is clearly a nonequilibrium and a highly nonlinear phenomenon.

Since the pioneer work by Cahn and Hilliard [21], phase separation in binary alloys has been studied extensively. As a result, we have almost understood the mechanism of coarsening processes. In the microscopic approach, Kawasaki dynamics [22] which involves exchanges between nearest-neighbor atoms. On the other hand, in a semi-phenomenological approach we use the time-dependent Ginzburg and Landau (TDGL) equation which is given by

$$
\frac{\partial S(\boldsymbol{r}, t)}{\partial t}=L \nabla^{2} \frac{\delta F}{\delta S(\boldsymbol{r}, t)}+\zeta(\boldsymbol{r}, t)
$$

where $S(r, t)$ is the local order parameter which is the local concentration difference between species A and B. We usually use a free energy functional $F\{S\}$ describing the excess energy of mixture

$$
F(\{S\})=\int d \mathbf{r}\left[\frac{1}{2}(\nabla S)^{2}+\frac{r_{0}}{2} S^{2}+u S^{4}-h S\right]
$$

where $r_{0}=\left(T-T_{0}\right) / T_{0}$ with the mean field critical temperature $T_{0}$ and $\boldsymbol{u}$ is a positive constant. The external field $h$ ensures the off-critical quench in which the minority phase has spherical forms (droplets). $\zeta(\boldsymbol{r}, t)$ represents a Gaussian thermal noise, which satisfies the fluctuation-dissipation theorem

$$
<\zeta(r, t)\rangle=0 \text { and }\left\langle\zeta(r, t) \zeta\left(r^{\prime}, t^{\prime}\right)>=-2 k_{B} T L \nabla^{2} \delta\left(r-r^{\prime}\right) \delta\left(t-t^{\prime}\right)\right.
$$

where $k_{B}$ is the Boltzmann constant. 
In contrast to the kinetic Ising model (Kawasaki dynamics) the derivation of TDGL equation contains a coarse-grained picture. When we discuss dynamics of phase transitions, the coarse-grained picture is more effective way[23] than microscopic pictures, because the correlation length is much larger than inter-atomic distances. In the late stage of phase separations, we can simplify the discussion using an interfacial approach [24] in which a further coarse-grained picture is used. In this stage the excess energy is accumulated in interface regions and dynamics can be characterized by the motion of interfaces. Kawasaki and Ohta[24] developed a systematic method to derive the interface equations of motion from TDGL equation.

In the late stage of phase separations one distinguishes between two different types of instability. The first is an instability against finite amplitude localized (droplet-like) fluctuation which is produced by a quench into the metastable region (off-critical quench). 'The second is an instability against infinitesimal amplitude, nonlocalized (long wave length) fluctuation which is produced by a quench into the unstable region (critical quench). The interfacial approach is applicable to both processes. Although we know that there is no sharp distinction between metastable and unstable states, the characteristics of dynamics is quite different with each other. In this thesis we mainly focus on the decay of metastable states, because coagulation of droplets plays an important role during coarsening processes.

In the final stage of the decay of a metastable state (off-critical quench) the growth of droplets due to condensation-evaporation process occurs, known as Ostwald ripening [25]. During the coarsening process the critical droplet radius grows with time where its growth law is represented by $R_{c}(t) \sim t^{1 / 3}$. In such a process small droplets shrink, while large droplets grow where mass transport takes place due to the Gibbs Thomson effects at 
droplets' surface. Lifshitz and Slyozov (and also Wagner) [25] analyzed this process based on a classical picture like Smoluchowski's coagulation theory. Recently, modern theories [26-28] have been developed. The niodern theories clarify the followings: (i) Ostwald ripening can be described by the equation of interfacial motion which is derived from TDGL equation (I.2.1) [23]. (ii) LSW (Lifshitz, Slyozov and Wagner) theory[25] is only valid for the dilute limit of volume fraction $\phi$. In reality, there is a correction term of the order $\phi^{(d-2) / 2}[26]$. (iii) There is the logarithmic dependence on the volume fraction for two dimensional Ostwald ripening $[27,28]$. The logarithmic correction which includes time does not disappear in finite time [28]. These results suggest that the classical theory in coagulation can not be applied to finite volume fraction case of droplets.

Kawasaki dynamics[24] and TDGL equation (I.2.1) contain a strong assumption in which atomic exchanges occur in a short distance as compared to the coarse-grained distance which defines the local order parameter. This assumption is satisfied in usual binary alloys, while it cannot be satisfied in more complex systems [29]. For instance, de Gennes[30] suggested that the transport coefficient has a nonlocal structure in phase separations of concentrated polymer mixture. Hayakawa and his colleague[31] proposed a generalization for a long-range exchange model. We can see a nonlocal effect in phase separation in binary fluid mixtures. Here a local concentration exerts a force which generates convective flow, which in turn, affects the local concentration a long distance away. Thus, we can regard this mechanism as an effective long-range exchange of fluid molecules. In fact, we can write down the equation for a viscous binary fluid mixture[32]

$$
\frac{\partial S(\boldsymbol{r}, t)}{\partial t}=-\int d \boldsymbol{r}^{\prime} W\left(\boldsymbol{r}, \boldsymbol{r}^{\prime} ;\{S\}\right) \frac{\delta F}{\delta S\left(\boldsymbol{r}^{\prime}, t\right)}+\zeta(\boldsymbol{r}, t)
$$


with

$$
\left\langle\zeta(\boldsymbol{r}, t)>=0 \text { and }\left\langle\zeta(\boldsymbol{r}, t) \zeta\left(\boldsymbol{r}^{\prime}, t^{\prime}\right)\right\rangle=-2 k_{B} T W\left(\boldsymbol{r}, \boldsymbol{r}^{\prime} ;\{S\}\right) \delta\left(\boldsymbol{r}-\boldsymbol{r}^{\prime}\right) \delta\left(t-t^{\prime}\right)\right. \text {. }
$$

For fluid mixtures $W$ is given by

$$
W\left(\boldsymbol{r}, \boldsymbol{r}^{\prime} ;\{S\}\right)=\nabla S(\boldsymbol{r})^{\prime} T\left(\boldsymbol{r}-\boldsymbol{r}^{\prime}\right) \nabla^{\prime} S\left(\boldsymbol{r}^{\prime}\right)-L \nabla^{2} \delta\left(\boldsymbol{r}-\boldsymbol{r}^{\prime}\right)
$$

where $T(\boldsymbol{r})$ is the Oseen tensor[33]

$$
T(r)=\frac{1}{8 \pi \eta} \frac{r^{2}+r r}{r^{3}}
$$

which incorporates a long-range exchange of molecules. In the case of a critical quench the characteristic length grows as $l(t) \sim 0.1(\sigma / \eta) t[11]$ where $\sigma$ and $\eta$ are the surface tension of interfaces and the viscosity of fluid, respectively. This growth law is complete different from the Lifshitz and Slyozov mechanism for solid [25]. Thus, we can expect more exotic feature in fluid mixtures than that in solid systems.

For the decay of a metastable state in solid systems we know that Ostwald ripening is the dominant process in the late stage of coarsening. Coagulation plays a role only in the middle stage of coarsening [10]. In off-critical quench of fluid mixtures the condensation evaporation process excited by fluid is canceled out[24]. Therefore, we cannot observe the growth law $l(t) \sim(\sigma / \eta) t$ like that in critical quenches. However, coalescence among droplets becomes important for fluid systems, because thermal fluctuation of liquid molecules produces the Brownian motion of liquid droplets. In the next section we show that the mean droplet radius grows $\bar{R}(t) \sim t^{1 / 3}$ with time for fluid mixtures. Although both growth laws for Ostwald ripening and for coagulation have the same time dependence, Siggia[11] pointed out that coagulation is the dominant process when the volume fraction 
of the minority phase (droplets) $\phi$ is larger than 0.01 . Ohta[34] also estimated that the cross over from Ostwald ripening to coagulation occurs at the $\phi=0.021$ by the comparison of the growth rates of mean droplet radius. Therefore, to investigate coagulation process is important for study of phase separations in fluid mixture.

\section{$\S$ I-3. Scaling Concepts in Coagulation}

In this section we introduce a key concept, dynamical scaling. we apply it to coagulation and extract essential feature of coagulation.

Dynamical scaling[10] is one of key concepts in nonequilibrium physical processes. There is a relevant length scale (the characteristic length ) in the system. If we rescale statistical quantities with the help of the characteristic length we obtain self-similar structure in rescaled quantities. For example, in a phase separation the interval between interfaces is the characteristic length and it grows with time obeying a power law of time. In the case of coagulation, the mean droplet radius is the characteristic length in the system. Even in the mean field type of kinetic equation (I.1.2), we know that the equations only for three types of rate constant, $K_{i j}=1[3], i+j[19]$ and $i j[7]$ in a certain unit, can be solved exactly (see Appendix B). Therefore, we cannot expect to get exact solutions in physical realistic situations. In such cases the concept of dynamical scaling gives a strong support understanding physical processes.

In literature various different forms for $K_{i j}$ are used to describe different physical processes. Simple examples of derivations of rate constants are presented in Appendix A. These different forms have one property in common, namely that always $K_{i j}$ is a 
homogeneous function of the sizes $i$ and $j$ [35]. For this reason we can express

$$
K(a i, a j)=a^{\lambda} K(i, j)
$$

for homogeneous kernels, where $\lambda$ is the degree of homogeneity. When we consider homogeneous kernels, the size distribution which has the scaling form

$$
c_{\mathrm{s}}(t)=M \bar{s}(t)^{-\tau} F(s / \bar{s}(t))
$$

where $M=\sum_{s} s c_{s}$ is the total mass of clusters or droplets and $\bar{s}(t)$ is the mean cluster size, satisfies the rate equation (I.1.2). It is easy to show that the exponent $\tau$ is $\tau=2$ in non-gelling systems, i.e. there is no singularities in moments of size, because $M$ is a constant in time. Note that in source-enhanced coagulation processes $[17,36,37]$ which lead to asymptotic power laws of size distribution the exponent $\tau$ is not equal to 2 due to the increase of total mass of clusters. Another important property is the power law growth of mean cluster size. Substituting (I.3.1) and (I.3.2) into (I.1.2) we obtain

$$
\bar{s}(t) \propto t^{1 /(1-\lambda)}
$$

for $-1<\lambda<1$. In the case of $\lambda=1$ like coagulation in shear flow the mean cluster size grows exponentially in time [5].

In this paper we focus on diffusion-controlled coagulation process. Smoluchowski[3] obtained the classical rate constant (Appendix A and section II-1) as

$$
K_{i j}=4 \pi D_{i j} R_{i j}
$$

for $\boldsymbol{d}=3$ where $D_{i j}=D_{i}+D_{j}$ is the relative diffusion constant and $R_{i j}=R_{i}+R_{j}$ is the reaction radius between $i$ and $j-$ mer. In the case of suspensions in fluid or binary fluid 
mixtures, the diffusion coefficient is given by $D_{i} \sim k_{B} T / \eta R_{i}$. Therefore, the growth law of mean cluster size is

$$
\bar{s}(t) \propto t
$$

because of $R_{s} \propto s^{1 / 3}$ and $D_{s} \propto R_{s}^{-1}$. The mean field theory discussed by Smoluchowski[3] can be generalized for $d$-dimensional systems, where the growth law (I.3.5) is valid because of $K_{i j} \propto D_{i j} R_{i j}^{d-2}$ and $D_{s} \propto R_{s}^{2-d}$.

\section{$\S$ I-4. Modern Theory of Coagulation: Beyond Smoluchowski's Theory}

In this section we explain trials for constructing a coagulation theory beyond Smoluchowski's theory[3].

It is clear that Smoluchowski's theory[3] is based on a kind of mean field idea, because it is assumed that the probability of coalescence is proportional to the concentrations of colliding clusters. Usually mean field theories are violated for low dimensional systems. Let us consider a one dimensional coagulation system as an example of violation of a mean field idea. It is clear that the coagulation rate is not proportional to the multiplication of mean concentrations of sizes of colliding clusters, because clusters collide their nearest-neighbor clusters. The validity of the mean-field theory is related to the recurrent probability of random walkers. In addition, Smoluchowski's approach does not contain spatial correlation and shape of droplets. 'The analysis for Ostwald ripening suggests that coagulation theory has a correction of finite density of droplets to the classical theory. It is natural to try to improve the classical theory by Sinoluchowski [3].

Several attempts have been carried out. One is to take into account fluctuation in size distribution in a finite system $[38,39]$. 'This method is based on the idea of van Kampen's 
(2-expansion method [40] which is a systematic expansion of the master equation in powers of inverse system size. Although Ernst and van Dongen[38] developed this method, one cannot consider the spatial correlation within their framework, which is more important in realistic systems. In addition, they regarded the coagulation kernel as an independent variable both of spatial correlation and dimension. This is not true. Therefore, it seems meaningless that van Dongen[39] suggested the violation of mean field theory even for $d \rightarrow \infty$.

The other approach is more realistic and effective. This approach is based on the Fock space formalism proposed by Doi[16], which is a formal second quantization of classical particles. The Fock space formalism[16,41] has been applied to many stochastic models such as the epidemic model[42], reaction-diffusion chemical processes including the contact process[43] and diffusion-controlled annihilations [44]. Hayakawa[12,17] generalized the original formalism to apply it to coagulation processes. In this approach we begin with the Liouville equation like that in gas dynamics. Our approach is equivalent to the Fokker Planck equation, namely, ours is a stochastic approach. The Liouville equation leads to the hierarchical equations for correlation functions like BBGKY hierarchy in usual many body problems. It is not diflicult to show that the critical dimension is two (see also [45]) , i.e. in systems for $d>2$ the classical theory is applicable in the dilute limit of density of clusters. However, Hayakawa[12] shows that the rate constant has finite density correction to Smoluchowski's classical one[3] even for $d=3$. We can discuss the size distribution and correlation function or structure factor (scattering function) using our approach.

In the next Chapter which is the central part of this paper we develop the coagulation theory using the Fock space formalism. We start from the Liouville equation to construct 
the hierarchical equations for correlation functions. Using a mean-field type approximation we derive the effective rate constant which is identical to Smoluchowski's one in the dilute limit and has correction of the order of $\phi^{1 / 2}$ for $d=3$, where $\phi$ is the volume fraction of droplets. From the straight-forward calculation we predict the scaling form for scattering function and compare it with an experimental result [18]. 


\section{References}

[1]. II.Sonntag and K.Strenge, Coagulation Kinetics and Structure Formation, (Plenum, New York 1987).

'I.Vicsek, Fractal Growth Phenomena (World Scientific, Singapore 1989).

R.Jullien and R.Botet, Aggregation and Fractal Aggregates (Singapore 1987).

P.G.J.van Dongen, Finetic Theory of Coagulation, (Phd.Thesis in Univ.of Utrecht, Utrecht 1987)

F.Family and D.P.Landau eds. Kinetics of Aggregation and Gelation (North-Holland, Amsterdam 1984).

[2]. B.B.Mandelbrot, The Fractal Geometry of Nature, (San Francisco 1982)

[3]. M.von Smoluchowski, Physik Z.17 (1916) 585.

M.von Smoluchowski, Z.Phys.Chem.92 (1918) 129.

[4]. S.Chandrasekhar, Rev.Mod.Phys. 15 (1943) 1.

[5]. S.K.Friedlander, Smoke, Dust and Haze(Wiley, New York 1977).

[6]. D.A.Weitz, J.S.Huang, M.Y.Lin and J.Sung, Phys.Rev.Lett. 54 (1985) 1396.

II.M.Lindsay, R.Klein, D.A.Weitz, M.Y.Lin and P.Meakin,Phys.Rev. A38 (1988) 2614 .

M.Y.Lin, H.M.Lindsay, D.A.Weitz, R.Klein, R.C.Ball and P.Meakin, J.Phys: Condens.Matter 2 (1990) 3093.

[7]. R.M.Ziff, J.Stat.Phys.23 (1980) 241.

R.M.Ziff, M.H.Ernst and E.M.Ilendriks, J.Phys.A16 (1983) 2293.

[8] G.B.Field and W.C.Saslaw, Astrophys.J. 142 (1965) 568.

C.IIayashi and Y.Nakagawa, Prog.Theor.Phys. 54 (1975) 93. 
H.Hayakawa and S.Hlayakawa, Publ.Astron.Soc.Jpn. 40 (1988) 341.

[9]. R.W.Samsel and A.S. Perelson, Biophys.J 37 (1982) 493.

[10]. K.Binder and D.Stauffer, Adv. Phys. 25 (1976) 343.

K.Binder, Ann.Phys. (New Y'ork) 98 (1976) 390.

[11]. E.D.Siggia, Phys.Rev.A20 (1979) 495.

[12]. II.Hayakawa, submitted to Physica $\mathbf{A}$ and Chapter II in this thesis.

[13]. P.Meakin, Phys.Rev.Lett. 51 (1983) 1119.

M.Kolb, R.Botet and R.Jullien, ibid 1123.

P.Meakin, in Phase Transitions and Critical Phenomena vol.12 C. Domb and J. L. Lebowitz eds. (Academic Press, London 1988)

[14]. F.Family, P.Meakin and J.M.Deutch, Phys.Rev.Lett. 57 (1986) 727.

P.G.J.van Dongen and M.H.Ernst, J.Stat.Phys. 37 (1984) 301.

M.H.Ernst and P.G.J. van Dongen, Phys.Rev.A36 (1987) 435.

[15]. H.Hayakawa, Mod.Phys.Lett. B2 (1988) 773.

[16]. M.Doi, J.Phys.A9 (1976) 1465,1479.

[17]. H.Hayakawa, J.Phys. A 22 (1989) 571.

[18]. C.M.Knobler and N.C.Wong, J.Phys.Chem. 85 (1981) 1972.

[19]. W.I.Scott, J.Atmos.Sci. 25 (1968) 54.

E.M.Hendriks, R.M.Ziff and M.H.Ernst, J.Stat.Phys. 31 (1983) 519.

[20]. J.D.Gunton and M.Droz, Introduction to the Theory of Metastable and Unstable States: Lecture Notes in Physics, vol.183 (Springer, Berlin, 1983).

J.D.Gunton, M.S.Miguel and P.S.Sahni, Phase Thansitions and Critical Phenomena vol.8 C.Domb and J.L.Lebowitz eds. (Academic Press, London 1983). 
H.Furukawa, Adv.Phys. 34 (1985) 703.

[21]. J.W.Cahn and J.E.IIilliard, J.Chem.Phys. 28 (1958) 258.

J.W.Cahn and J.E.Hilliard, J.Chem.Phys. 31 (1959) 688.

[22] K.Kawasaki, Phys.Rev. 145 (1966) 224; 148 (1966) 375; 150 (1966) 285.

K.Kawasaki, Phase Transitions and Critical Phenomena vol.2 C.Domb and M.S.Green eds. (Academic Press, London 1972).

[23]. Y.Oono and S.Puri, Phys.Rev.Lett. 58 (1987) 836; introduce the more effective method to simulate coarsening processes.

[24]. K.Kawasaki and 'T.Ohta, Physica 118A (1983) 175.

[25] I.M.Lifshitz and V.V.Slyozov, J.Phys.Chem.Solids 19 (1961) 35.

C.Wagner, Z.Electrochem. 65 (1961) 581.

[26]. J.A.Marqusee and J.Ross, J.Chem.Phys. 81 (1984) 976.

M.Tokuyama and K.Kawasaki, Physica 123A (1984) 386.

M.Marder, Phys.Rev.36A (1987) 858.

[27]. J.A.Marqusee, J.Chem.Phys.81(1984) 976.

Q.Zheng and J.D.Gunton, Phys.Rev.A39 (1989) 4848.

[28]. H.Hlayakawa and F.Family, Physica 163A (1990) 491.

[29]. K.Kawasaki and T.Koga, Prog.Theor.Phys.Suppl. 99 (1989) 339.

[30]. P.G.de Gennes, J.Chem.Phys.72 (1980) 4756.

[31]. H.Ilayakawa and F.Family, Physica 166A (1990) 408.

II.Hlayakawa, T.Koga and F.Family, in Dynamics and Patterns in Complex Fluids A.Onuki ed. (Springer, Berlin 1990).

II.Hlayakawa and 'T.Koga, J.Phys.Soc.Jpn. 59 (1990) 3542. 
[32]. K.Kawasaki, Physica 119A (1983) 17.

[33]. see e.g. M.Doi and S.F.Edwards, The Theory of Polymer Dynamics, ( Oxford Univ. Press, Oxford 1986 ).

[34]. 'T.Ohta, Ann.Phys.(New Y'ork) 163 (1984) 31.

[35]. P.G.J.van Dongen and M.II.Ernst, Phys.Rev.Lett. 54 (1985) 1396.

P.G.J.van Dongen and M.II.Ernst, J.Stat.Phys.50 (1988) 295.

[36]. H.'Takayasu, I.Nishikawa and II.'Tasaki, Phys.Rev. A37 (1988) 3110.

H.Takayasu Phys.Rev.Lett.63 (1989) 2563.

H.'Takayasu, M.'Takayasu, A.Provata and G.Huber, submitted to J.Stat.Phys.

[37]. II.Ilayakawa, M.Yamamoto and II.Takayasu, Prog.Theor.Phys. 78 (1987) 1.

H.Ilayakawa, J.Phys. A20 (1987) L801.

[38]. P.G.J.van Dongen and M.II.Ernst, J.Stat.Phys.49 (1987) 879.

P.G.J.van Dongen, J.Stat.Phys. 49 (1987) 927; 53 (1988) 221; 54 (1989) 221.

[39]. P.G.J.van Dongen, Phys.Rev.Lett. 63 (1989) 1281 ; J.Stat.Phys.58 (1990) 87.

[40]. N.G. van Kampen, Stochastic Processes in Physic and Chemistry (North-Holland, Amsterdam 1981).

[41]. Ya.B.Zel'dovich and A.A.Ovchinnikov, Pis'ma Zh.Eksp. Teor. Fiz. 26 (1977) 588 [JE'TP Lett.26 (1977) 440].

P.Grassberger and M.Scheunert, Fortschr.Phys.28 (1980) 547.

L.Peliti, J.Physique 46 (1985) 1469.

[42]. J.L.Cardy, J.Phys.A16 (1983) L709.

J.L.Cardy and P.Grassberger, J.Phys.A18 (1985) L267.

[43]. P.Grassberger and K.Sundermeyer, Phys.Lett.77B (1978) 220. 
'T.Ohtsuki and 'T.Keyes, Phys.Rev. A35 (1987) 2697.

[44]. A.S.Mikhailov and V.V.Yashin, J.Stat.Phys.38, (1985) 347.

A.M.Gutin, A.S.Mikhailov and V:V.Yashin, Zh. Eksp. Teor. Fiz. 92 (1987) 941 [Sov. Phys. JETP.65 (1987) 533].

[45] L.Peliti, J.Phys.A19 (1986) L365.

D.Elderfield, J.Phys.A20 (1987) L135. 


\title{
Chapter II. Fluctuation Effects in Coagulation Processes
}

\begin{abstract}
of this chapter
We study diffusion-controlled coagulation processes in the system of finite density of droplets using the Fock space formalism. We find that the effective reaction rate has correction to Smoluchowski's classical rate constant for three dimensional systems. We also predict the scaling form for the scattering function and find that our results qualitatively agree with experimental results.
\end{abstract}




\section{$\S$ II-1. Introduction}

Coagulation [1] is an irreversible process in which basic units (monomers) stick together to form clusters or droplets. This process takes place in many fields of science such as polymer physics[2], colloid chemistry[3], aerosol physics[4,5], phase separations [6-8] and astrophysics[9]. In contrast to simulations[10] and experiments[1,3] theoretical description, basically, remains at the classical level by Smoluchowski[11]. Several attempts[12-14] clarify that the classical theory for diffusion-controlled coagulation is valid for three dimensional systems in dilute limit. The classical theory, however, does not contain the spatial correlations and the validity of the classical theory for finite density cases is not clear. Therefore, we need a modern theory of coagulation processes.

Let us consider a phase separation of binary liquids [15]. In the low volume fraction case, the time evolution of phase separation is determined by the competition between Ostwald ripening[16] and Brownian coagulation. Siggia[6] pointed out that coagulation is the dominant process when the volume fraction of droplets $\phi$ is larger than 0.01 . Although several authors $[7,8,17]$ predicted scaling forms for the scattering function and their volume fraction dependence for binary fluid mixtures, it seems that these theories remain at phenomenological and classical levels. We should construct a theory for coagulation processes from the first principle.

First, we give a brief explanation of the classical theory. Let us consider the situation that a test droplet of $i$-mer which consists of $i$ monomers stays at the origin and coalescence process takes place between $i$-mer and $j$-mer. Let $w_{i j}(r, t)$ be the concentration of $j$-mer at a point $r$ relative to the test droplet of $i$-mer. Smoluchowski[11] assumed that coalescence 
process can be described by the diffusion equation

$$
\frac{\partial w_{i j}}{\partial t}=D_{i j} \nabla^{2} w_{i j}
$$

where $D_{i j}=D_{i}+D_{j}$ is the relative diffusion constant. The boundary condition is

$$
\begin{gathered}
w_{i j}(\boldsymbol{r}, t=0)=c_{i} \quad \text { for } \quad r \equiv|r|>R_{i j} \equiv R_{i}+R_{j} \\
w_{i j}(\boldsymbol{r}, t)=0 \quad \text { at } \quad r=R_{i j} .
\end{gathered}
$$

Since the flux of $i$-mer arriving at the reaction surface $r=R_{i j}$ is proportional to the gradient of $w_{i j}(r, t)$ at the reaction surface, the flux current is given by

$$
J_{i j}=4 \pi D_{i j} R_{i j} c_{i}
$$

for $t \gg R_{i j}^{2} / D_{i j}$. We assume that the time evolution of size distribution is governed by a simple mass balance equation

$$
\frac{\partial c_{s}(t)}{\partial t}=\frac{1}{2} \sum_{i+j=s} J_{i j} c_{j}-c_{s} \sum_{j=1}^{\infty} J_{s j} .
$$

This equation is well-known as Smoluchowski's rate equation [11]. Smoluchowski[11] solved the rate equation with the approximation $D_{i j} R_{i j} \sim$ const. Friedlander and Wang[5] adopt the scaling ansatz

$$
c_{s}(t)=\phi \bar{s}(t)^{-2} F_{3}(s / \bar{s}(t)),
$$

where $\bar{s}(t)$ is the mean droplet size and $F_{3}(x)$ is the three dimensional scaling function for droplet size distribution. They numerically solved (II.1.3),(II.1.4) and (II.1.5) and found the followings: (i) Smoluchowski's approximation which is equivalent to $F_{3}(x)=e^{-x}$ is not bad for large cluster sizes, while the scaling function tends to zero for $x \rightarrow 0$. (ii) The mean droplet radius $\bar{R}$ and the number density of droplets $\rho$ obey power laws in time as 


$$
\bar{R}(t) \sim t^{1 / 3} \quad \text { and } \quad \rho \sim t^{-1}
$$

where they assumed that coagulation occurs in liquids. For fluid mixtures the diffusion coefficient $D_{\boldsymbol{i}}$ is given by

$$
D_{i}=\frac{k_{B} T}{5 \pi \eta R_{i}}
$$

where $T$ is the temperature, $k_{B}$ is the Boltzmann constant, and $\eta$ is the viscosity of the background fluid. The classical theory predicts $J_{i j} \sim D_{i j} R_{i j}^{d-2} c_{i}$ for a $d$-dimensional system where the diffusion coefficient for a fluid system is scaled as $D_{i j} \sim R_{i j}^{2-d}$.

Although the classical theory seems plausible, there are several critical questions. The above approach is a kind of effective two-body approximation in which reactions among surrounding droplets are not taken into account. Such an approximation is analogous to LSW (Lifshitz, Slyozov and Wagner) theory[16] in Ostwald ripening. As well-known[18-20] LSW theory is only valid for dilute limit for three dimensional systems, while a screening length plays an important role for finite density systems. In addition, Smoluchowski's theory cannot be applied to two dimensional coagulation [12-14,21]. In this case the finiteness of number density of droplets is essential. Therefore, we should consider the finite density effect in coagulation processes.

In this chapter we show the the mass current $J_{i j}$ has correction due to finite density. For spherical droplets for $d=3$ the explicit form of the current is given by

$$
J_{i j}=4 \pi D_{i j} R_{i j} c_{j}\left[1+\sigma_{i j} R_{i j}\right]
$$


where

$$
\sigma_{i j}^{2}=\frac{2 \pi}{D_{i j}} \sum_{l=i, j} \sum_{m} D_{l m} R_{l m} c_{m}
$$

is the square of inverse of the screening length. From the result of (II.1.8) and (II.1.9), it is easy to obtain the scaling form for scattering function and its volume fraction dependence. We compare our theoretical results with experimental results for fluid mixtures.

The arrangement of this chapter is as follows. In section II-2 we formulate coagulation processes based on the Fock space formalism which is a method to describe dynamics of classical particles. In section II-3 we develop a perturbative expansion using the diagrammatic method. In section II-4 we obtain the effective coagulation rate and show that the effect of finite density of droplets is important in both two and three dimensional systems. In section II-5 we predict the scaling form of the scattering function based on the droplet picture. We compare our results with experiments for binary fluid mixtures[15] and previous phenomenological theories[7,8,17]. In the final section we present concluding remarks.

\section{$\S$ II-2. Basic Formalism}

In this section we show the outline of the Fock space formalism [22-25]. Doi[22] has proposed the Fock space formalism which is a formal second quantization procedure to describe various stochastic processes. In particular, diffusion controlled reaction processes $[12,22,26,27]$ are good subjects for this formalism. Hayakawa[14] generalized the original formulation to apply it to coagulation processes. Our stochastic approach [14] is applicable to any dimensional systems even in $d=1$. Here, we present the formulation based on the argument in Ref.[14]. We assume that each droplet has a spherical form 
and the relaxation from a collision of two droplets to a large spherical droplet is fast. We neglect the backflow effects of fluids during the relaxation.

In our formalism the state vector of each size of droplets is independent. Therefore the state vector $\mid \Phi(t)>$ is normal product of the state vector of each size as $|\Phi(t)\rangle=$ $\otimes_{\mathbf{s}}\left|\Phi_{s}(t)\right\rangle$. The time evolution of a state vector $|\Phi(t)\rangle$ is described by

$$
\frac{\partial \mid \Phi(t)>}{\partial t}=L \mid \Phi(t)>
$$

where $L$ is the Liouvillian and it consists of two parts as

$$
L=L_{0}+L_{\text {int }}
$$

'The free part $L_{0}$ in (II.2.2a) represents diffusion

$$
L_{0}=\sum_{s} \int d r a_{s}^{\dagger}(r) D_{s} \nabla^{2} a_{s}(r)
$$

with the diffusion coefficient $D_{s}$ of $s$-mer, while the interactive part $L_{i n t}$ describes the coagulation process

$$
L_{i n t}=\frac{1}{2} \sum_{i, j} \int d r d r^{\prime} K_{i j}\left(r-r^{\prime}\right)\left\{a_{i+j}^{\dagger}\left(\frac{i r+j r^{\prime}}{2(i+j)}\right) a_{i}(r) a_{j}\left(r^{\prime}\right)-a_{i}^{\dagger}(r) a_{j}^{\dagger}\left(r^{\prime}\right) a_{i}(r) a_{j}\left(r^{\prime}\right)\right\}
$$

In (II.2.2) $a_{s}^{\dagger}(r)$ and $a_{s}(r)$ are creation and annihilation operators, which satisfy the Bose commutation relations

$$
\left[a_{i}(r), a_{j}\left(r^{\prime}\right)\right]=\left[a_{i}^{\dagger}(r), a_{j}^{\dagger}\left(r^{\prime}\right)\right]=0 \quad \text { and } \quad\left[a_{i}(r), a_{j}^{\dagger}\left(r^{\prime}\right)\right]=\delta_{i j} \delta\left(r-r^{\prime}\right)
$$

For simplicity we assume that the reaction rate is given by

$$
K_{i j}(r)=K_{0} \theta\left(R_{i j}-|r|\right)
$$


where $\theta(t)$ is the step function, that is, $\theta(t)=1$ for $t>0$ and $\theta(t)=0$ for $t<0$. In our model reactions occur within the capture radius $R_{i j}$.

In this formalism the number density of $s$-mer and the pair correlation function between $i$-mer and $j$-mer are respectively given by

$$
c_{s}(r, t)=<\operatorname{sum}\left|a_{s}^{\dagger}(r) a_{s}(r)\right| \Phi(t)>/ V \equiv<a_{s}^{\dagger}(r) a_{s}(r)>/ V=<a_{s}(r)>/ V,
$$

where $V$ is the volume of the system, and

$$
g_{i j}\left(r, r^{\prime}, t\right)=<a_{i}^{\dagger}(r) a_{j}^{\dagger}\left(r^{\prime}\right) a_{i}(r) a_{j}\left(r^{\prime}\right)>/ V^{2}=<a_{i}(r) a_{j}\left(r^{\prime}\right)>/ V^{2} .
$$

In (II.2.5) and (II.2.6) the state $\mid$ sum $>$ is produced from the null (vacuum) state $\mid 0>$ as

$$
\left|\operatorname{sum}>=\exp \left\{\sum_{i} \int d r a_{i}^{\dagger}(r)\right\}\right| 0>
$$

To obtain the final expressions in (II.2.5) and (II.2.6) we have used

$$
a_{s}(\boldsymbol{r}) \mid \text { sum }>=\mid \text { sum }>\text {. }
$$

From (II.2.1) to (II.2.8) we obtain the time evolution of the number density of droplets as

$$
\begin{gathered}
\frac{\partial c_{s}(\boldsymbol{r}, t)}{\partial t}=D_{s} \nabla^{2} c_{s}(\boldsymbol{r}, t)+\frac{1}{2} \sum_{i+j=s} \int d \boldsymbol{r}^{\prime} K_{i j}\left(\boldsymbol{r}-\boldsymbol{r}^{\prime}\right) g_{i j}\left(\boldsymbol{r}, \boldsymbol{r}^{\prime}, t\right) \\
-\sum_{j=1}^{\infty} \int d \boldsymbol{r}^{\prime} K_{s j}\left(\boldsymbol{r}-\boldsymbol{r}^{\prime}\right) g_{s j}\left(\boldsymbol{r}, \boldsymbol{r}^{\prime}, t\right) .
\end{gathered}
$$

Similarly $g_{i j}\left(\boldsymbol{r}, \boldsymbol{r}^{\prime}, t\right)$ obeys the equation which contains the three body correlation function. These equations are analogous to the BBGKY hierarchy in usual many body problems.

Equation (II.2.9) corresponds to Smoluchowski's rate equation. Since we are interested in the spatial homogeneous case, $c_{\boldsymbol{s}}(\boldsymbol{r}, t)$ and $g_{i j}\left(\boldsymbol{r}, \boldsymbol{r}^{\prime}, t\right)$ are respectively written as $c_{\boldsymbol{s}}(t)$ and $g_{i j}\left(r-r^{\prime}, t\right)$. 'The classical theory is the result of the approximation

$$
\int d r^{\prime} K_{i j}\left(r-r^{\prime}\right) g_{i j}\left(r-r^{\prime}, t\right)=4 \pi D_{i j} R_{i j} c_{i}(t) c_{j}(t)
$$


for $d=3$. Although the validity of this approximation in dilute limit cases has been already discussed, we do not know whether (II.2.10) is still valid in finite density cases. In the proceeding discussion we show that (II.2.10) should have correction when we consider the systems in finite density of droplets.

Let us rewrite (II.2.9) as

$$
\frac{\partial c_{s}(t)}{\partial t}=\frac{1}{2} \sum_{i+j=s} K_{i j}^{e f f} c_{i}(t) c_{j}(t)-\sum_{j=1}^{\infty} K_{s j}^{e f f} c_{s}(t) c_{j}(t)
$$

for the spatial homogeneous case. 'The effective reaction rate $K_{i j}^{e f f}$ satisfies

$$
K_{i j}^{e f f}=\int d r K_{i j}(r) f_{i j}(r)
$$

where $f_{i j}(r)$ is identical to $w_{i j}(r) / c_{i}$ in section II-1 and is defined by

$$
f_{i j}(r, t)=\frac{g_{i j}(r, t)}{c_{i}(t) c_{j}(t)}=1+h_{i j}(r, t)
$$

where the spatial correlation disappears a long distance away, i.e., $h_{i j}(r \rightarrow \infty) \rightarrow 0$. Thus, the problem is reduced to obtaining $f_{i j}(r)$.

The correlation function $f_{i j}(r)$ or $h_{i j}(r)$ is also an important quantity to predict the functional form of the scattering function. Ohta[7] presented the formula for the scattering function $I_{q}(t)=I_{|\vec{q}|}(t)$

$$
\begin{aligned}
I_{q}(t) & =\sum_{s} s^{2} c_{s}(t) \Psi_{d}\left(q R_{s}\right)^{2}+\sum_{i, j} i j c_{i}(t) c_{j}(t) \Psi_{d}\left(q R_{i}\right) \Psi_{d}\left(q R_{j}\right) \int d r\left[f_{i j}(r, t)-1\right] e^{i \vec{q} \vec{r}} \\
& =\sum_{s} s^{2} c_{s}(t) \Psi_{d}\left(q R_{s}\right)^{2}+\sum_{i, j} i j c_{i}(t) c_{j}(t) \Psi_{d}\left(q R_{i}\right) \Psi_{d}\left(q R_{j}\right) h_{i j}(q)
\end{aligned}
$$

where $i=(4 / 3) \pi R_{\mathrm{i}}^{3}$ for $d=3$ and $i=\pi R_{\mathrm{i}}^{2}$ for $d=2$. $i \Psi_{d}(x)$ is the structure factor for a single spherical droplet of $i$-mer in $d$-dimensional space and its form is

$$
\Psi_{3}(x)=3 \frac{\sin x-x \cos x}{x^{3}}
$$


for $d=3$ and

$$
\Psi_{2}(x)=\frac{2}{x} J_{1}(x)
$$

for $d=2$, where $J_{1}(x)$ is the Bessel function. In (II.2.14) $h_{i j}(q) \equiv h_{i j}(|q|)$ is the Fourier component of $h_{i j}(r)$ and has relations

$$
h_{i j}(r)=V^{-1} \sum_{\vec{q}}^{\prime} h_{i j}(q) e^{i \vec{q} \vec{r}} \quad \text { and } \quad h_{i j}(q)=\frac{<a_{i}(q) a_{j}(-q)>}{V c_{i}(t) c_{j}(t)} .
$$

The prime on the summation in (II.2.16) indicates that the summation is performed over all $k$ except for $k \equiv|k|=0$. To obtain the scattering function we need to know both $h_{i j}(q)$ and $c_{i}(t)$. In the derivation of (11.2.14) we neglect the overlapping of droplets. Therefore, (II.2.14) is valid for the volume fraction $\phi \ll \phi_{c}$ where $\phi_{c}$ is the percolation threshold of the minority phase.

\section{$\S$ II-3. The Diagrammatic Perturbation}

In this section we develop the perturbative expansion to calculate $K_{i j}^{e f f}$ and $f_{i j}(r)$. Using the Fourier transform as

$$
a_{s}(k)=\int d r e^{i \vec{k} \vec{r}} a_{s}(r),
$$

(II.2.9) can be rewritten as

$$
\frac{\partial c_{s}(t)}{\partial t}=\frac{1}{2 V^{2}} \sum_{\vec{k}}\left\{\sum_{i+j=s} K_{i j}(k)<a_{i}(k) a_{j}(-k)>-2 \sum_{j=1}^{\infty} K_{s j}(k)<a_{s}(k) a_{j}(-k)>\right\} .
$$

For simplicity we use the same notations both in the real space and Fourier space.

Introducing the interaction representation with $\tilde{a}_{\boldsymbol{s}}(k, t)=\exp \left(-L_{0} t\right) a_{\boldsymbol{s}}(k) \exp \left(L_{0} t\right)$ we have the Liouville equation

$$
\frac{\partial \mid \tilde{\Phi}(t)>}{\partial t}=\tilde{L}_{\text {int }}(t) \mid \tilde{\Phi}(t)>
$$


where $\tilde{L}_{i n t}(t)=\exp \left(L_{0} t\right) L_{i n t} \exp \left(-L_{0} t\right)$ and $\left|\tilde{\Phi}(t)>=\exp \left(L_{0} t\right)\right| \Phi(t)>$. Thus the correlation in (II.3.2) can be rewritten as

$$
\begin{aligned}
<a_{i}(k) a_{j}(-k)>= & <\operatorname{sum}\left|\tilde{a}_{i}(k, t) \tilde{a}_{j}(k, t) U\left(t, t_{0}=0\right)\right| \tilde{\Phi}\left(t_{0}=0\right)> \\
& \equiv<\tilde{a}_{i}(k, t) \tilde{a}_{j}(k, t) U(t, 0)>_{0}
\end{aligned}
$$

with the evolution operator

$$
U\left(t, t_{0}\right)=\hat{T} \exp \left[\int_{t_{0}}^{t} L_{\text {int }}\left(t^{\prime}\right) d t^{\prime}\right]
$$

where $\hat{T}$ is the time ordering operator. The method for the perturbation is analogous to that in finite temperature systems for solid state physics. We perform the perturbative expansion by expanding $U(t, 0)$ into the products of the propagator which is defined through

$$
\begin{array}{r}
<\hat{T} \tilde{a}_{i}^{\dagger}(k, t) \tilde{a}_{j}\left(k^{\prime}, t^{\prime}\right)>_{0}=\delta_{i j} \delta\left(k-k^{\prime}\right) G_{i}^{0}\left(k-k^{\prime}, t-t^{\prime}\right) \\
=\delta_{i j} \delta\left(k-k^{\prime}\right) \exp \left(-D_{i} k^{2}\left(t-t^{\prime}\right)\right) \theta\left(t-t^{\prime}\right)
\end{array}
$$

where $\theta(t)$ is the step function.

We summarize the Feymmann rule (see Figs.II-1 and II-2) on the perturbation expansion as follows. 'The thin line (Fig.II-1(a)) is the free propagator where the arrow represents the direction of time. The zigzag line (Fig.II-1(b)) corresponds to the "condensate" number of $s$-mer $N_{s}(t)=<a_{s}(k=0)>$ and the dashed line (Fig.II-1(c)) is $1=<a_{s}^{\dagger}(k=0)>$. There are two kinds of bare vertices, where the three point vertex (Fig.II-2(a)) is $\Gamma_{i j \rightarrow i+j}^{0}\left(k, k^{\prime} ; k+k^{\prime}\right) / 2 V=K_{i j}^{\prime}(k) / 2 V$ and the four point vertex (Fig.II2(b)) is $\Gamma_{i j \rightarrow i j}^{0}(\boldsymbol{p}, \boldsymbol{q} ; \boldsymbol{p}+k, \boldsymbol{q}-k) / 2 V=-K_{i j}(k) / 2 V$. At the vertices the momentum and frequency are conserved. Thus, the problem of obtaining $K_{i j}^{\text {eff }}$ is reduced to the calculation 
of the correction of the three point vertex function $\Gamma_{i j \rightarrow i+j}(k)$ (Fig.II-2(c)). Note that a four point vertex is identical to a three point vertex except for its sign when one of legs is $<a_{s}^{\dagger}(0)>=1$. We count irreducible diagrams in terms of the standard rule to calculate the correction to the vertex. Let us note that there are only retarded propagators and no branched vertices, because the propagator vanishes for $t<0$.

\section{Figure II-1 - Figure II-2}

In the previous paper[14] we show that the classical theory which corresponds to the ladder approximation is valid in dilute limit. Here we discuss the volume fraction dependence on the effective reaction rate $K_{i j}^{\text {eff }}$, where we adopt the self-consitent meanfield approximation in the level of the ladder. In this approximation the real propagator of droplets (Fig.II-1(d)) defined in

$$
G_{i j}\left(k, t-t^{\prime}\right)=<\hat{T} \tilde{a}_{i}^{\dagger}(k, t) \tilde{a}_{j}\left(k, t^{\prime}\right) U(t, 0)>_{0}
$$

obeys the Dyson equation as

$$
G_{i j}(k, z)=G_{i}^{0}(k, z) \delta_{i j}+\sum_{m} G_{i}^{0}(k, z) \Sigma_{i m}(k, z) G_{m, j}(k, z)
$$

where $G(k, z)=\int_{0}^{\infty} e^{-z t} G(k, t) d t$ and $\Sigma_{i m}(k, z) \simeq \Sigma_{i m}(0,0)$ is the self-energy. The real propagator and the self-energy have, in general, nondiagonal $(i, j)$ elements [14], because coagulation during its propagation makes the size of droplet increasing.

In low density case we can neglect nondiagonal terms from the following reasons. Let us consider a three dimensional system. In low density case the self-energy (Fig.II-3) can be represented as

$$
\Sigma_{i j}(0,0) \simeq\left[c_{j-i} K_{i, j-i}^{c l}-\delta_{i j} \sum_{m} c_{m} K_{i m}^{c l}\right] / 2
$$


where $K_{i j}^{c l} \equiv 4 \pi D_{i j} R_{i j}$ is the classical coagulation rate calculated by Smoluchowski[11]. It is clear that the value of the second term which exists only in diagonal elements is much larger than that of the first term in the right hand side of (II.3.9), because the summation runs over all possible sizes. Since we can ignore nondiagonal terms in the self-energy and we only consider the reaction of $(i, j)$ pair, the problem is essentially reduced to the problem of the diffusion-controlled pair annihilation process[22,26]. It is interesting that in the case of diffusive annihilation with two kinds of particles off-diagonal terms are important [27], while they are irrelevant for coagulation cases. 'The situation in two dimensional systems is similar to that in three dimension. The value of the diagonal elements for three point vertex function which contains the summation of all possible sizes is much larger than that of the off-diagonal one. Therefore, the contribution from off-diagonal elements to vertex functions is negligible.

\section{Figure II-3 - Figure II-4}

The effective coagulation rate is obtained in the integral equation for the vertex function $\Gamma_{i j}(k) \equiv \Gamma_{i j \rightarrow i+j}(k,-k ; 0)$ which satisfies (see Fig.II-4)

$$
\Gamma_{i j}(k) \simeq K_{i j}(k)-\frac{1}{D_{i j}} \int \frac{d k^{\prime}}{(2 \pi)^{d}} \frac{K_{i j}\left(k-k^{\prime}\right) \Gamma_{i j}\left(k^{\prime}\right)}{k^{2}+\sigma_{i j}^{2}}
$$

where

$$
\sigma_{i j}^{2}=\frac{\left|\Sigma_{i i}(0,0)\right|+\left|\Sigma_{j j}(\boldsymbol{O}, 0)\right|}{D_{i j}}+\frac{z}{D_{i j}}
$$

Note that $\sigma_{i j}^{-1}$ is the screening length of diffusion field of $(i, j)$ pair. This approximation is identical to the Hartree-Fock approximation in quantum mechanics. In the limit of $k \rightarrow 0$ we obtain the reaction rate. From $\left(\right.$ II.2.16) $h_{i j}(k)$ is related to the vertex function as

$$
h_{i j}(k)=-\frac{\Gamma_{i j}(k)}{D_{i j}\left(k^{2}+\sigma_{i j}^{2}\right)}
$$


Therefore, to obtain $\Gamma_{i j}(k)=\Gamma_{i j}(|k|)$ is a key problem.

\section{$\S$ II-4. The Effective Reaction Rate}

We now solve the self-consistent equation (II.3.10). After the inverse Fourier transform (II.3.10) can be rewritten as

$$
\Gamma_{i j}(r)=K_{i j}(r)-D_{i j}^{-1} K_{i j}(r) Z_{i j}(r)
$$

where $K_{i j}(r)=K_{i j}(|r|)$ is given in (II.2.4) and $Z_{i j}(r)$ is defined by

$$
Z_{i j}(r)=\int \frac{d k}{(2 \pi)^{d}} \frac{\Gamma_{i j}(k) e^{i \vec{k} \vec{r}}}{k^{2}+\sigma_{i j}^{2}}
$$

Note that $Z_{i j}(r)$ satisfies

$$
\left(\nabla^{2}-\sigma_{i j}^{2}\right) Z_{i j}(r)=K_{i j}(r)\left[D_{i j}^{-1} Z_{i j}(r)-1\right]
$$

From now on, we present the analysis both for $d=3$ and $d=2$.

\section{(a). Three Dimensional Case}

First we calculate the effective reaction rate in a three dimensional system. The spherical symmetric solution of (II.4.3) for $r<R_{i j}$ is

$$
Z_{i j}(r)=A_{3} \frac{\sinh \left(\mu_{i j} r\right)}{r}+\frac{K_{0}}{\mu_{i j}^{2}}
$$

where $A_{3}$ is the constant detemined by (II.3.11) and $\mu_{i j}^{2}=\sigma_{i j}^{2}+K_{0} / D_{i j}$. Thus, the integral in (II.3.10) is carried out as 


$$
\begin{gathered}
\Gamma_{i j}(k)=\frac{4 \pi K_{0} \sigma_{i j}^{2}}{\mu_{i j}^{2} k^{3}}\left[\sin \left(k R_{i j}\right)-k R_{i j} \cos \left(k R_{i j}\right)\right] \\
-\frac{4 \pi K_{0} A_{3}}{D_{i j} k\left(k^{2}+\mu_{i j}^{2}\right)}\left[\mu_{i j} \cosh \left(\mu_{i j} R_{i j}\right) \sin \left(k R_{i j}\right)-k \sinh \left(\mu_{i j} R_{i j}\right) \cos \left(k R_{i j}\right)\right] .
\end{gathered}
$$

From (II.4.4) and (II.4.5) with the low density condition $\sigma_{i j} R_{i j} \ll 1$, we obtain the constant $A_{3}$ as

$$
A_{3} \simeq-\frac{D_{i j} R_{i j}}{\lambda_{i j} \cosh \lambda_{i j}}\left[1+\sigma_{i j} R_{i j}\left(1-\frac{\tanh \lambda_{i j}}{\lambda_{i j}}\right)\right]
$$

in the order of $\sigma_{i j} R_{i j}$, where

$$
\lambda_{i j}^{2} \equiv \frac{K_{0} R_{i j}^{2}}{D_{i j}} \simeq\left(\mu_{i j} R_{i j}\right)^{2}
$$

represents the strength of reaction. In this approximation the first term in the right hand side of (II.4.5) is negligible. Hence the effective reaction rate is reduced to

$$
K_{i j}^{e f f}=\Gamma_{i j}(k=0)=4 \pi D_{i j} R_{i j}\left[1+\sigma_{i j} R_{i j}\left(1-\frac{\tanh \lambda_{i j}}{\lambda_{i j}}\right)\right]\left[1-\frac{\tanh \lambda_{i j}}{\lambda_{i j}}\right],
$$

where $\sigma_{i j}$ is given by

$$
\sigma_{i j}^{2}=\frac{2 \pi}{D_{i j}} \sum_{l=i, j} \sum_{m} D_{l m} R_{l m} c_{m}\left[1-\frac{\tanh \lambda_{i j}}{\lambda_{i j}}\right]+\frac{z}{D_{i j}}
$$

Equations(II.4.8) and (II.4.9) contain familiar results. For rapid coagulation $\left(\lambda_{i j} \gg 1\right)$ (II.4.8) is reduced to

$$
K_{i j}^{e f f}=4 \pi D_{i j} R_{i j}\left[1+\sigma_{i j} R_{i j}\right]
$$

where

$$
\sigma_{i j}^{2}=\frac{2 \pi}{D_{i j}} \sum_{l=i, j} \sum_{m} D_{l m} R_{l m} c_{m}+\frac{z}{D_{i j}} .
$$

For fluid systems the first term in the right hand side of (II.4.10a) $\left(\sigma_{(1)}^{2}\right)$ is much larger than the second term $\left(\sigma_{(2)}^{2}\right)$, because the first term can be estimated as $\sigma_{(1)}^{2} \sim \rho \bar{R}(t) \sim t^{-2 / 3}$ 
while the second term is $\sigma_{(2)}^{2} \sim 1 / t$, where we have used (II.1.6) and (II.1.7). From now on, we neglect the second term in (II.4.9) and (II.4.10b) when we discuss three dimensional coagulation. In zero density limit $\left(\sigma_{i j} R_{i j} \ll 1\right)$, (II.4.10) is identical to Smoluchowski's result. On the other hand, if a droplet of radius $R$ is immobile except for monomers (diffusion coefficient $D$ ), i.e. $R_{l m} \simeq R_{m}$ and $D_{l m}=D_{i j}=D$, the reaction rate (II.4.10) is reduced to

$$
K(R)=4 \pi D R[1+R \sqrt{4 \pi \rho \bar{R}(t)}]
$$

This expression is identical to that for Ostwald ripening obtained by Marqusee and Ross [18]. Note that the expression in (11.4.10) can be obtained by the extension of conventional theory (Appendix A).

For the slow reaction $\left(\lambda_{i j} \ll 1\right)$ we reproduce the chemical kinetics

$$
K_{i j}^{e \rho \rho}=\frac{8 \pi}{3} K_{0} R_{i j}^{3}
$$

which corresponds to chemical kinetic process. Note that (II.4.12) is independent of the volume fraction, at least, in the level of the present approximation. The reaction rate in (II.4.12) is equivalent to that in the shear flow[4], where $K_{0}$ corresponds to the shear rate (Appendix A). 'Therefore, the mean droplet size $\bar{s}(t)$ grows as $\bar{s}(t) \sim \exp \left[K_{0} \phi t\right]$ with time. It is interesting to compare our result with the experimental results for the reaction limited aggregation (RLA) of colloidal particles. In experimental situations, colloidal aggregates form fractal clusters. 'Then small clusters can stay in the fjord region of a large cluster where the distance between the centers of mass of two clusters is shorter than the reaction radius. Although the cluster shape is restricted to the sphere in our model, the overlapping of clusters is permitted for slow reactions because of the definition of the reaction rate 
(1I.2.4). 'This situation is similar to that in the experimental one for RLA, where we observe the exponential growth of mean cluster size[28] as time goes on.

The functional form of the correlation function $h_{i j}(r)$ or $h_{i j}(k)$ in (II.2.13) can be derived from (II.3.14) and (II.4.5). We obtain the pair correlation function $h_{i j}(k)$ in (II.3.12) as

$$
h_{i j}(k)=-\frac{4 \pi K_{0}\left[1+\sigma_{i j} R_{i j}\left(1-\tanh \lambda_{i j} / \lambda_{i j}\right)\right]}{D_{i j} k\left(k^{2}+\sigma_{i j}^{2}\right)\left(k^{2}+K_{0} / D_{i j}\right)}\left[\sin \left(k R_{i j}\right)-\frac{k R_{i j} \tanh \lambda_{i j} \cos \left(k R_{i j}\right)}{\lambda_{i j}}\right] .
$$

In the rapid coagulation case $h_{i j}(k)$ is reduced to

$$
h_{i j}(k) \simeq-\frac{4 \pi \sin \left(k R_{i j}\right)}{k\left(k^{2}+\sigma_{i j}^{2}\right)} .
$$

Then the real part $h_{i j}(r)=h_{i j}(|v|)$ is given by

$$
h_{i j}(r) \simeq-\frac{\exp \left(-\sigma_{i j} R_{i j}\right)}{\sigma_{i j} r} \sinh \left(\sigma_{i j} r\right)
$$

for $r<R_{i j}$ and

$$
h_{i j}(r) \simeq-\frac{\exp \left(-\sigma_{i j} r\right)}{\sigma_{i j} r} \sinh \left(\sigma_{i j} R_{i j}\right)
$$

for $r>R_{i j}$. Note that $h_{i j}(r) \rightarrow-1$ for $r<R_{i j}$ and $h_{i j}(r) \rightarrow-R_{i j} / r$ for $r>R_{i j}$ in low density limit. Our results ensure that the correlation disappears within the reaction region. These results correspond to the classical argument by Smoluchowski[11].

\section{(b). Two Dimensional Case}

Now we discuss the eflective reaction rate in two dimension. Although the procedure is parallel to that in three dimensional case, we cannot use the density expansion in two dimensional case. Therefore, the effective reaction rate can be determined only in a selfconsistent form. 
We come back to (II.4.3). 'The spherical symmetric solution for $r<R_{i j}$ is

$$
Z_{i j}(r)=A_{2} I_{0}\left(\mu_{i j} r\right)+\frac{K_{0}}{\mu_{i j}^{2}}
$$

where $A_{2}$ is a constant and $I_{0}(x)$ is the first-order modified Bessel function. 'Thus, the integration of (II.3.10) is

$$
\begin{gathered}
\Gamma_{i j}(k)=\frac{2 \pi\left(\sigma_{i j} R_{i j}\right)^{2} J_{1}\left(k R_{i j}\right)}{k \mu_{i j}^{2} R_{i j}} \\
-\frac{2 \pi K_{0} A_{2}}{D_{i j}\left(k^{2}+\mu_{i j}^{2}\right)}\left[k R_{i j} J_{1}\left(k R_{i j}\right) I_{0}\left(\mu_{i j} R_{i j}\right)+\mu_{i j} R_{i j} J_{0}\left(k R_{i j}\right) I_{1}\left(\mu_{i j} R_{i j}\right)\right],
\end{gathered}
$$

where $J_{1}(x)$ and $J_{0}(x)$ are the Bessel functions and $I_{1}(x)$ is the first order modified Bessel function. In the low density case, $\sigma_{i j} R_{i j} \ll 1$ and $\mu_{i j} R_{i j} \simeq \lambda_{i j}$, we obtain the constant $A_{2}$ as

$$
A_{2} \simeq-\frac{D_{i j}}{\lambda_{i j} I_{1}\left(\lambda_{i j}\right)\left|\ln \left(\sigma_{i j} R_{i j}\right)\right|}
$$

Substituting (II.4.19) into (II.4.18) we obtain the effective reaction rate

$$
K_{i j}^{e f f}=\frac{2 \pi D_{i j}}{\left|\ln \left(\sigma_{i j} R_{i j}\right)\right|},
$$

where $\sigma_{i j}$ satisfies a self-consistent condition

$$
\sigma_{i j}^{2}=\frac{\pi}{D_{i j}} \sum_{l=i, j} \sum_{m} \frac{D_{l m} c_{m}(t)}{\left|\ln \left(\sigma_{l m} R_{l m}\right)\right|}+\frac{z}{D_{i j}} .
$$

In this case the both terms in the right hand side of (II.4.21) are the same order. Due to the existence of the second term the scaling is violated and has the logarithmic correction through the reaction rate in (II.4.20). These results are also generalization of results in two dimensional Ostwald ripening[29].

From (II.4.18) and (II.4.19) we obtain $h_{i j}(k)$ as

$$
h_{i j}(k)=-\frac{2 \pi\left[k R_{i j} J_{1}\left(k R_{i j}\right) I_{0}\left(\lambda_{i j}\right)+\lambda_{i j} J_{0}\left(k R_{i j}\right) I_{1}\left(\lambda_{i j}\right)\right]}{\lambda_{i j} I_{1}\left(\lambda_{i j}\right)\left|\ln \left(\sigma_{i j} R_{i j}\right)\right|\left(k^{2}+\sigma_{i j}^{2}\right)} .
$$


Note that the derivation of (II.4.20),(II.4.21) and (II.4.22) are independent of strength of the reaction defined in (II.4.7). For rapid coagulation cases $\left(\lambda_{i j} \gg 1\right)$, the contribution from the first term in the numerator in (II.4.22) is negligible, because the first order modified Bessel functions have asymptotic forms $I_{1}\left(\lambda_{i j}\right) \sim I_{0}\left(\lambda_{i j}\right) \sim \exp \left(\lambda_{i j}\right) / \sqrt{2 \pi \lambda_{i j}}$ for $\lambda_{i j} \gg 1$. Therefore $h_{i j}(k)$ is reduced to

$$
h_{i j}(k) \simeq-\frac{2 \pi J_{0}\left(k R_{i j}\right)}{\left|\ln \left(\sigma_{i j} R_{i j}\right)\right|\left(k^{2}+\sigma_{i j}^{2}\right)} .
$$

The real part of the correlation function has the form

$$
h_{i j}(r) \simeq-\frac{I_{0}\left(\sigma_{i j} R_{i j}\right) K_{0}\left(\sigma_{i j} r\right)}{\left|\ln \left(\sigma_{i j} R_{i j}\right)\right|}
$$

for $r>R_{i j}$ and

$$
h_{i j}(r) \simeq-\frac{I_{0}\left(\sigma_{i j} r\right) K_{0}\left(\sigma_{i j} R_{i j}\right)}{\left|\ln \left(\sigma_{i j} R_{i j}\right)\right|}
$$

for $r<R_{i j}$. In low density limit, $h_{i j}(r)$ has an asymptotic form $h_{i j}(r) \simeq-\frac{K_{0}\left(\sigma_{i j} r\right)}{\left|\ln \left(\sigma_{i j} R_{i j}\right)\right|}$ for $r>R_{i j}$ and $h_{i j}(r) \simeq-1$ for $r<R_{i j}$. These results ensure no correlation within the reaction region.

\section{$\S$ II-5. Scaling in the Scattering Function}

In this section we calculate the scaling form for the scattering function and compare our results with an experimental results[15].

Dynamical scaling is one of key concepts in nonequilibrium physics. In the late stage of phase separation and the coagulation of suspensions, dynamical scaling laws are often observed. Adopting the scaling ansatz[5,30] of the size distribution of droplets (II.1.5) is rewritten as

$$
c_{s}(t)=\phi \bar{s}(t)^{-2} F_{d}(s / \bar{s}(t) ; \phi),
$$


where $\bar{s}(t)$ is the mean droplet size. The scaling function $F_{d}(x ; \phi)$, in general, depends on the spatial dimension $d$ and the volume fraction of droplets $\phi$ which is defined by $\phi=\sum_{s} s c_{s}(t)$. We assume that the scaling function $F_{d}(x)$ satisfies the normalization conditions as

$$
\int_{0}^{\infty} F_{d}(x ; \phi) d x=\int_{0}^{\infty} x F_{d}(x ; \phi) d x=1
$$

The correlation function also can be scaled as

$$
h_{i j}(q)=-\phi^{-1} \bar{s}(t) H_{d}(Q ; x, y)
$$

with $Q=q[3 \bar{s}(t) / 4 \pi]^{1 / 3}$ for $d=3 . H_{d}(Q ; x, y)$ in (II.5.3) is the scaling function where $x$ and $y$ are $x=i / \bar{s}(t)$ and $y=j / \bar{s}(t)$, respectively. The factor $\phi^{-1}$ comes from $h_{i j}(q) \propto \phi$ in the limit $q \rightarrow 0$. For simplicity, we restrict our interest to the three dimensional rapid coagulation case in the proceeding discussions.

Substituting (II.5.1) and (II.5.3) into (II.2.14) we obtain the scattering function for $d=3$

$$
I_{q}(t)=\phi(1-\phi) \bar{R}(t)^{3} \hat{I}_{3}(Q),
$$

where $\bar{R}=[3 \bar{s}(t) / 4 \pi]^{1 / 3}$ is the mean droplet radius. The factor $\phi(1-\phi)$ comes from the normalization condition of the scattering function

$$
\phi(1-\phi)=\int \frac{d q}{(2 \pi)^{3}} I_{q}(t)
$$

From (II.2.14) the scaling function $\hat{I}_{3}(Q)$ in (II.5.4) is given by

$$
\hat{I}_{3}(Q)=[\Theta(Q)-\chi \Xi(Q)] \frac{1}{1-\phi}
$$

where $\chi$ is the adjustable constant to satisfy $\hat{I}_{3}(Q) \rightarrow 0$ for $Q \rightarrow 0$ because of the total conservation law. Strictly speaking, the scattering function satisfies $I_{q}(t) \rightarrow \bar{s}(t=0)$ for 
$q \rightarrow 0$, which is not equal to zero and the scaling is violated in low $q$ region. However, we can approximate $\hat{I}_{3}(Q \rightarrow 0) \simeq 0$ taking into account the power law growth of mean droplet size. From (II.5.4) and (II.5.5) it is easy to show that the scaling function $\hat{I}_{3}(Q)$ satisfies

$$
\int \frac{d Q}{(2 \pi)^{3}} \hat{I}_{3}(Q)=1
$$

From (II.2.14) and (II.5.1) $\Theta(Q)$ in (II.5.6) is given by

$$
\Theta(Q)=\frac{4 \pi}{3} \int_{0}^{\infty} d x x^{2} F_{3}(x ; \phi) \Psi_{3}\left(x^{1 / 3} Q\right)^{2}
$$

where $\Psi_{3}(x)$ is defined in (II.2.15a), while the functional form of $\Xi(Q)$ in (II.5.6) is given by

$$
\Xi(Q)=\frac{4 \pi}{3} \int_{0}^{\infty} d x \int_{0}^{\infty} d y x y F_{3}(x ; \phi) F_{3}(y ; \phi) \Psi_{3}\left(x^{1 / 3} Q\right) \Psi_{3}\left(y^{1 / 3} Q\right) H_{3}(Q ; x, y),
$$

Substituting the functional forms of $\Psi_{3}(x), F_{3}(x ; \phi)$ and $H_{3}(Q ; x, y)$ into (II.5.6), (II.5.8) and (II.5.9) we obtain the scaling function $\hat{I}_{3}(Q)$ of (II.5.6). If we obtain the exact form of $H_{3}(Q ; x, y)$ and $F_{3}(x ; \phi)$ the adjustable parameter $\chi$ should satisfy $\chi=1$. Therefore, to check the value of $\chi$ is a good test of the validity of our treatment.

'To obtain the scattering function we need to know the functional forms of $H_{3}(Q)$ in (II.5.3) and $F_{3}(x ; \phi)$ in (II.5.1). In the system of binary liquid mixture the diffusion coefficient in three dimensional systems is given by (II.1.7). Since we are interested in the low volume fraction case, we neglect the fraction effect in the size distribution function $\epsilon(x ; \phi)$ where $F_{3}(x ; \phi)=F_{3}(x ; 0)+\epsilon(x ; \phi)$. This approximation may be justified because the pair correlation function $h_{i j}$ contains the effect of $\phi$ and $\epsilon(x ; \phi)$ is the higher order correction of $\phi$ in (II.5.9). For the classical model Friedlander and Wang[5] numerically obtain

$$
\bar{s}(t) \simeq \frac{4 k_{B} T}{5 \eta} C \phi t \quad \text { with } \quad C \simeq 2.13
$$


and an interpolation formula $[5,7]$ for the scaling function for size distribution. The explicit form of the interpolation formula for $F_{3}(x ; 0)$ is given by

$$
F_{3}(x ; \phi=0)=\frac{0.915\left(x^{1.06}+0.5558\right)}{x^{1.06}} \exp \left[-0.95 x-b_{1} x^{2 / 3}+b_{2} x^{1 / 3}-1.275 x^{-1 / 3}\right]
$$

with

$$
\begin{array}{ll}
b_{1}=0.9754, & b_{2}=2.009 \quad \text { for } x<0.5 \\
b_{1}=0.9963, & b_{2}=1.897 \quad \text { for } x>0.5 .
\end{array}
$$

The expression (II.5.11) almost recovers the original numerical result in Ref.[5]. We introduce the moment of size distribution as

$$
M_{\alpha}=\int_{0}^{\infty} d x x^{\alpha} F_{3}(x ; 0)
$$

where the normalization conditions (II.5.2) satisfies with $98 \%$ accuracy, i.e. $M_{0} \simeq 0.98$ and $M_{1} \simeq 1.00[8]$.

The functional form of $H_{3}(Q ; x, y)$ in (II.5.3) is determined by (II.4.13). Since we are interested in a diffusion controlled case, we restrict our interest to the case for $\lambda_{i j} \gg 1$. From the scaling ansatz it is easy to show

$$
R_{i j}=\left(\frac{3 \bar{s}(t)}{4 \pi}\right)^{1 / 3}\left(x^{1 / 3}+y^{1 / 3}\right)
$$

where $x=i / \bar{s}(t)$ and $y=j / \bar{s}(t)$. From (II.4.10) and (II.1.7) the self-energy can be scaled as

$$
\sigma_{i j}^{2}=\phi\left[\frac{3}{4 \pi} \bar{s}(t)\right]^{-2 / 3} \nu(x, y)
$$

where the scaling function $\nu(x, y)$ is

$$
\nu(x, y)=3 \frac{(x y)^{1 / 3}}{x^{1 / 3}+y^{1 / 3}}\left[4+M_{1 / 3}\left(x^{-1 / 3}+y^{-1 / 3}\right)+M_{-1 / 3}\left(x^{1 / 3}+y^{1 / 3}\right)\right]
$$


with $M_{1 / 3} \simeq 0.90$ and $M_{-1 / 3} \simeq 1.23$. Substituting (II.5.13) and (II.5.14) into (II.4.13) with the help of (II.5.3) we obtain

$$
H_{3}(Q ; x, y) \simeq \frac{3 \phi \sin \left[Q\left(x^{1 / 3}+y^{1 / 3}\right)\right]}{Q\left[Q^{2}+\phi \nu(x, y)\right]}
$$

When we compare our result in (II.5.16) with the previous discussions $[7,8]$, our expression contains the size dependence which was neglected in the previous theories.

The adjustable parameter $\chi$ in (II.5.6) is determined by the condition $I_{3}(Q \rightarrow 0) \rightarrow 0$, which is equivalent to

$$
M_{2}=3 \chi \int_{0}^{\infty} d x \int_{0}^{\infty} d y \frac{x y F_{3}(x) F_{3}(y)\left(x^{1 / 3}+y^{1 / 3}\right)}{\nu(x, y)}
$$

where we use $\Psi_{3}(x \rightarrow 0) \rightarrow 1$. Substituting (II.5.11) into (II.5.17)and performing the numerical integral we find that the fitting parameter has the value

$$
\chi \simeq 1.68
$$

where we use $M_{2} \simeq 1.94$. The above result is far from the desired result $\chi=1$. The discrepancy mainly arises from the size distribution function (II.5.11) which is an approximated form for $\phi \rightarrow 0$. In fact, if we adopt the approximation $F_{3}(x)=e^{-x}$ as in Ref.[8], the fitting parameter can be estimated as $\chi \simeq 2.13$ which is worse than (II.5.18).

The final expression of the scaling form of the scattering function is obtained from the numerical integration of (II.5.7) and (II.5.9) with the help of (II.5.11) and (II.5.16). Instead of the scaling function $\hat{I}_{3}(Q)$ defined in (II.5.6) we use $S(x)$ which is defined through

$$
\hat{I}_{3}(Q)=Q_{m}^{-3} S\left(Q / Q_{m}\right)
$$

where $Q_{m}$ is the peak position of the scattering function. 


\section{Figure II-5}

We show the scaling function $S(x)$ for several values of $\phi$ in Fig.II-5. When the volume fraction increases, the function $S(x)$ becomes higher and narrower. Such properties can be seen in Figs.II-6 and II-7 for the peak height and the full width of half maximum, respectively. Our scaling results are similar to those obtained by Ohta[7] and Tomita[8] which were based on the Brownian coagulation model.

\section{Figure II-6 - Figure II-7}

Figure II- 8 displays the moment ratio $\left\langle x^{2}\right\rangle /\langle x\rangle^{2}$, where $\left\langle x^{n}\right\rangle$ is given by

$$
<x^{n}>=\frac{\int_{0}^{\infty} d x x^{n} S(x)}{\int_{0}^{\infty} d x S(x)} .
$$

We also compare our results with the experiment by Knobler and Wong[15]. In our results the shape of scaling function is broader than that obtained by the experiment. The experimental data is normalized in the interval from $x=0.3$ to $x=3.0$. Therefore, the discrepancy between our theory and the experiment is not serious.

\section{Figure II-8 - Figure II-9}

Our scaling function satisfies the Porod's law (Fig.II-9) for large wave number. The tail is dominated by the single droplet part $\Theta(Q)$ in (II.5.8). This tail part is related to the interface area density. Noting $\Theta(Q) \gg \Xi(Q)$ for $Q \gg 1$ we can estimate $\hat{I}_{3}(Q) \simeq$ $12 \pi Q^{-4} \int_{0}^{\infty} x^{2 / 3} F_{3}(x ; 0) \cos ^{2}\left(x^{1 / 3} Q\right) \sim 17 / Q^{4}$ where we use $<x^{2 / 3}>\sim 0.91$ and $\overline{\cos ^{2}(z)}=$ 1/2. Note that our theory contains the oscillatory tail as for the monodisperse case[17].

\section{§ II-6. Concluding Remarks}

In this chapter which is the central part of this thesis, we formulate the coagulation process using the Fock space formalism. We find that the screening length plays an impor- 
portant role in coagulation processes. Therefore, the correction to Smoluchowski's theory is important. In the low volume fraction case, the correction due to the finite density can be treated as a small perturbation for $d=3$, while the logarithmic correction does not disappear for $d=2$. These situations are common in all kinds of diffusion controlled reaction such as Ostwald ripening [18-20,29].

Our theoretical work is far from complete, because our theory contains a fitting parameter to predict the scaling form for the scattering function. In addition, we do not obtain quantitative agreement with the experiment. The problem arises from the following three points. (i) First, we neglect the volume fraction dependence of size distribution during our calculation of the scattering function. In the scattering function the contribution from the correction to the size distribution may not be small. The correction can be obtained by a numerical method and the results will be discussed elsewhere. (ii) The second, we use the mean-field approximation to obtain the effective reaction rate. Although we obtain the correction of the order $\phi^{(d-2) / 2}$, our result may not be exact in this order. In Ostwald ripening there is contribution from the soft collision process $[19,20]$. We believe there is the effects from soft collision even in coagulation systems. The soft collision enhances the scattering function in Ostwald ripening[31]. The discrepancy between the experiment and our theory may be improved when we consider the soft collision effect. (iii) Finally, in experimental situations of binary fluid mixtures, there is competition between Ostwald ripening and the Brownian coagulation. When we compare our results with the experiment, the effects from Ostwald ripening should be considered. Recently, Enomoto and Okada[32] have presented an interesting paper in which they discuss the size distribution of droplets in the competition between Ostwald ripening and Brownian coagulation. We 
need to develop their arguments furthermore. In future we should improve the points mentioned above and construct a more reasonable theory.

In conclusion, we present the statistical theory of Brownian coagulation based on the Fock space formalism. We obtain the finite density correction to Smoluchowski's classical theory. Using our formalism we predict the scaling form for the scattering function. Our results qualitatively agree with the experimental results for binary fluid mixture.

\section{Acknowledgment}

The author would like to thank Prof.K.Kawasaki and Dr.T.Kawakatsu for helpful discussions and useful suggestions. The author expresses his sincere gratitude to Prof. H. Tomita for his useful comments. 'The author thanks the warm hospitality during his stay at the Yukawa Institute for Theoretical Physics at Kyoto where the part of this work was carried out. This work is partially supported by the Fellowships of the Japan Society for the Promotion of Science for Japanese Junior Scientists. 


\section{References}

[1]. H.Sonntag and K.Strenge, Coagulation Kinetics and Structure Formation, (Plenum, New York 1987).

'I.Vicsek, Fractal Growth Phenomena (World Scientific, Singapore 1989).

[2] R.M.Ziff, J.Stat.Phys.23 (1980) 241.

F.C.Mackintosh, S.A.Safran and P.A.Pincus, Europhys.Lett. 12 (1990) 697.

[3] M.L.Broide and R.J.Cohen, Phys.Rev.Lett.64 (1990) 2026.

M.Doi and D.Chen, J.Chem.Phys.90 (1989) 5271.

M.Doi and D.Chen, J.Chem.Phys.91 (1989) 2656.

H.M.Lindsay, R.Klein, D.A.Weitz, M.Y.Lin and P.Meakin,Phys.Rev. A 38 (1988) 2614.

M.Y.Lin, H.M.Lindsay, D.A.Weitz, R.Klein, R.C.Ball and P.Meakin, J.Phys: Condens.Matter 2 (1990) 3093.

[4] F.S.Lai, S.K.Friedlander, J.Colloid Interface Sci. 39, 395 (1972).

S.K.Friedlander, Smoke, Dust and Haze(Wiley, New York 1977).

[5] S.K.Friedlander and C.S.Wang, J.Colloid Interface Sci. 22 (1966) 126.

[6] E.D.Siggia, Phys.Rev.A20 (1979) 495.

[7] 'T.Ohta, Ann.Phys.(New York) 163 (1984) 31.

[8] H.'Tomita, Prog.'Theor.Phys.71 (1984) 1405; the discrepancy of the value of moments between his paper ('Table I) and ours comes from the normalization where he used $M_{0}=1$.

[9] G.B.Field and W.C.Saslaw, Astrophys.J. 142 (1965) 568.

C.Ilayashi and Y.Nakagawa, Prog.Theor.Phys. 54 (1975) 93. 
H.Hayakawa and S.Hayakawa, Publ.Astron.Soc.Jpn. 40 (1988) 341.

[10] P.Meakin, Physica 165A (1990) 1 and references therein.

[11] M.von Smoluchowski, Physik Z.17 (1916) 585.

M.von Smoluchowski, Z.Phys.Chem.92 (1918) 129.

[12] L.Peliti, J.Phys.A19 (1986) L365.

[13] D.Elderfield, J.Phys.A20 (1987) L135.

[14] I.Ilayakawa, J.Phys.A22 (1989) 571.

[15] C.M.Knobler and N.C.Wong, J.Phys.Chem. 85 (1981) 1972.

[16] I.M.Lifshitz and V.V.Slyozov, J.Phys.Chem.Solids 19 (1961) 35.

C.Wagner, Z.Electrochem. 65 (1961) 581.

[1i] P.A.Rikvold and J.D.Gunton, Phys.Rev.Lett. 49 (1982) 286.

[18] J.A.Marqusee and J.Ross, J.Chem.Phys. 81 (1984) 976.

[19] M.'Tokuyama and K.Kawasaki, Physica 123A (1984) 386.

[20] M.Marder, Phys.Rev.36A (1987) 858.

[21] P.G.J.van Dongen, Phys.Rev.Lett. 63 (1989) 1281 ; J.Stat.Phys.58 (1990) 87; suggested that the upper critical dimension may not be two for some cases of coagulation.

[22] M.Doi, J.Phys.A9 (1976) 1465,1479.

[23] Ya.B.Zel'dovich and A.A.Ovchinnikov, Pis'ma Zh.Eksp. Teor. Fiz. 26 (1977) 588 [JETP Lett.26 (1977) 440].

[24] P.Grassberger and M.Scheunert, Fortschr.Phys.28 (1980) 547.

[25] L.Peliti, J.Physique 46 (1985) 1469.

[26] A.S.Mikhailov and V.V.Yashin, J.Stat.Phys.38, (1985) 347.

[27] A.M.Gutin, A.S.Mikhailov and V.V.Yashin, Zh. Eksp. Teor. Fiz. 92 (1987) 941 [Sov. 
Phys. JETP.65 (1987) 533].

[28] R.C.Ball, D.A.Weitz, 'I.A.Witten and F.Leyvraz, Phys.Rev.Lett. 58 (1987) 274.

[29] H.Hayakawa and F.Family, Physica163A (1990) 491.

[30] P.G.J.van Dongen and M.II.Ernst, J.Stat.Phys.50 (1988) 295.

[31] M.'Tokuyama, Y.Enomoto and K.Kawasaki, Physica 143A (1987) 183.

[32] Y.Enomoto and A.Okada, J.Phys: Condens.Matter 2 (1990) 4531. 


\section{Figure Captions}

Fig.II-1 The Feynmann diagrams for propagators; (a) the free propagator, (b) the zigzag line which corresponds to the number of droplet $N_{s}(t)=<a_{s}(0, t)>$, (c) $<a_{s}^{\dagger}(t)>=1$, and (d) the real propagator which contains the effect of interaction.

Fig.II-2 The Feynmann diagrams for vertices; (a) the three point bare vertex function, (b) the four point bare vertex function, and (c) the renormalized vertex function.

Fig.II-3 'The Dyson equation where the renormalized vertex is calculated in the zero density limit of droplets.

Fig.II-4 The self-consistent equation (II.3.9) where the renormalized vertex is determined by Fig.II-3 and thick lines are the real propagators.

Fig.II-5 'The volume fraction dependence of scaled scattering function. The solid line, the dashed line and the broken line correspond to the results of $\phi=0.03, \phi=0.08$ and $\phi=0.15$, respectively.

Fig.II-6 The volume fraction dependence of the peak height of scaled scattering function $y=$ $S(x)$. 'The curves (a),(b),(c) and (d) represent the theoretical predictions in the present thesis, Ref.[7], Ref[8] and Ref.[17]. The vertical lines display the experimental results in Ref.[15]. The method for the normalization of experimental data and theoretical curves follows that in Ref.[8].

Fig.II-7 The volume fraction dependence of the full width of half maximum of the scaled scattering function $S(x)$. The details are the same as in Fig.II-6.

Fig.II-8 The volume fraction dependence of the moment ratio $\left\langle x^{2}\right\rangle /\langle x\rangle^{2}$ which defined in (II.5.20). The curves (a),(b) and (c) correspond to the theoretical results by the present theory, Ref.[7] and Ref.[17]. The vertical lines show the experimental 
results[15].

Fig.II-9 The Porod's plot of the scaled scattering function $S(x)$, where the Porod function is given by $y=x^{4} S(x)$. 'The flat part represents the interfacial density. 


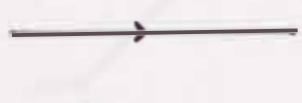

(a)
MMOMW

(b)

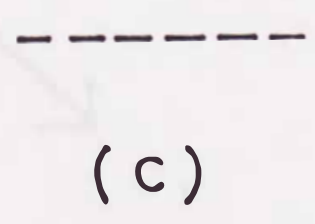

(c)

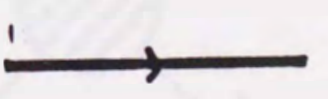

(d)

Fig.II-1 

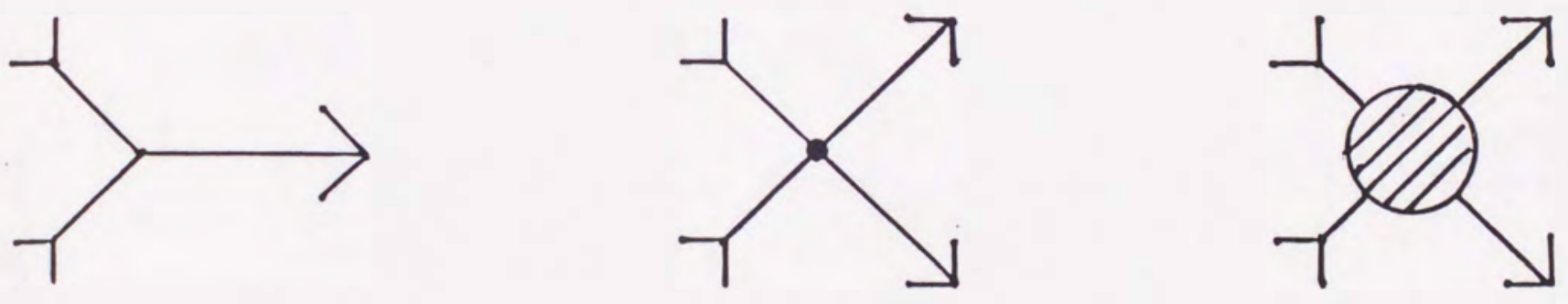

(a)

(b)

(c)

Fig.II-2 


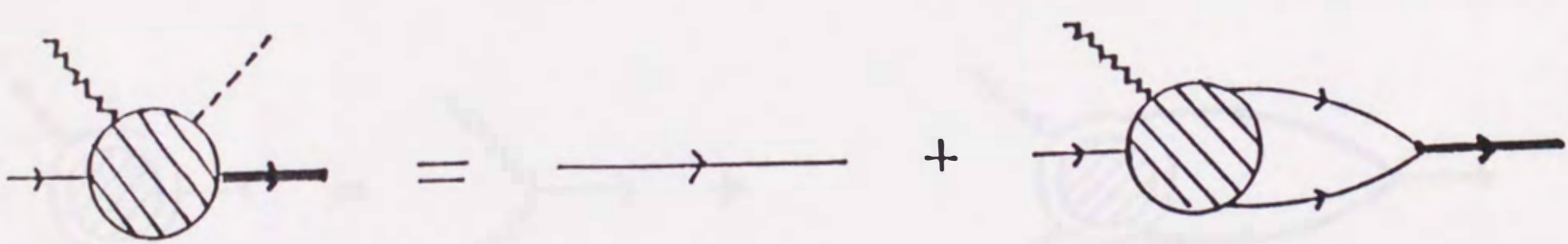

Fig.II-3 

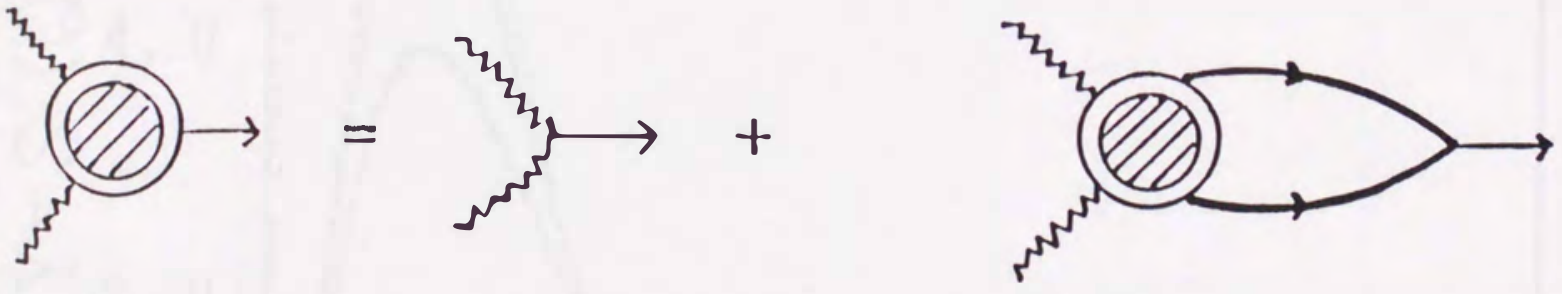

Fig.II-4 


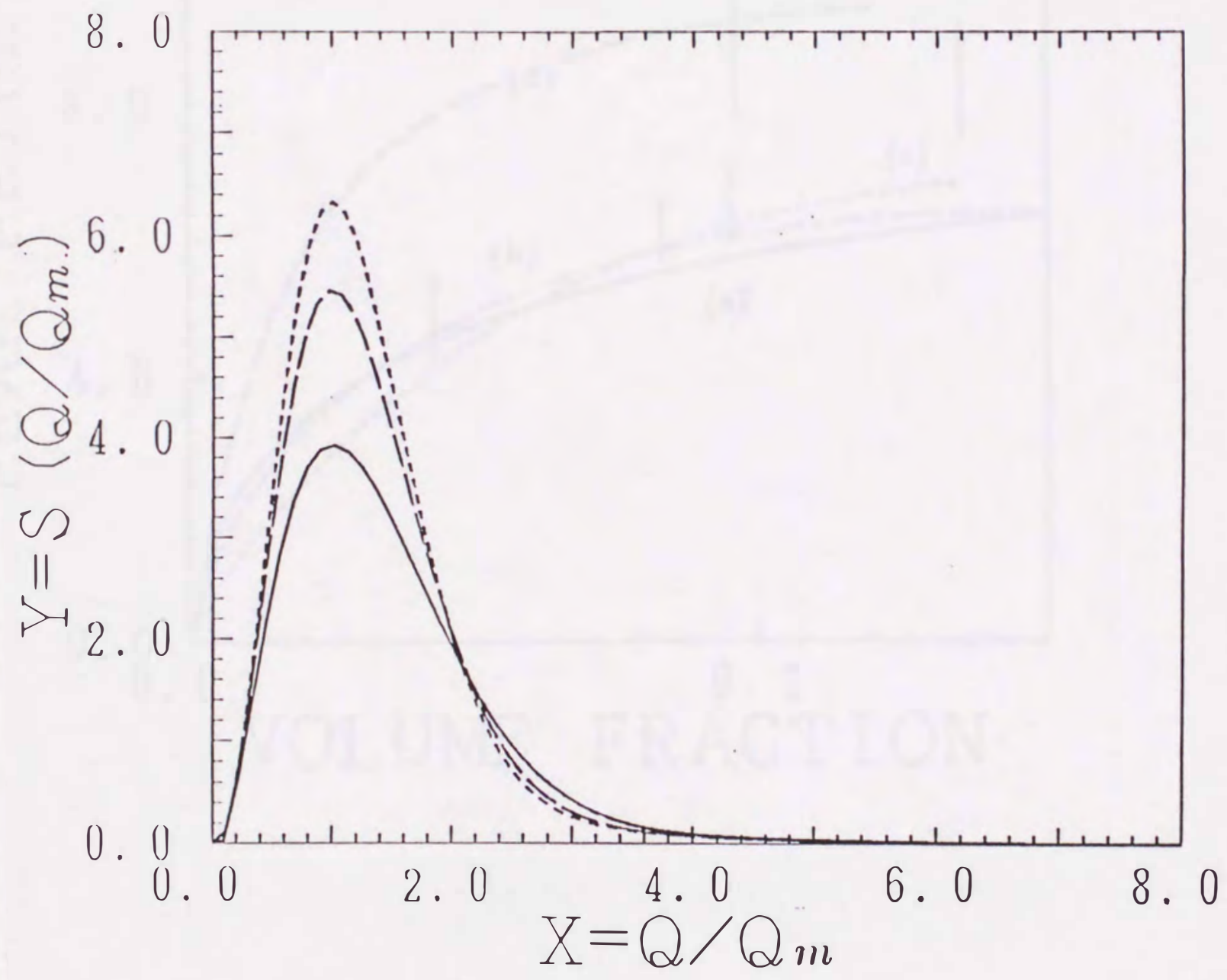

Fig.II-5 


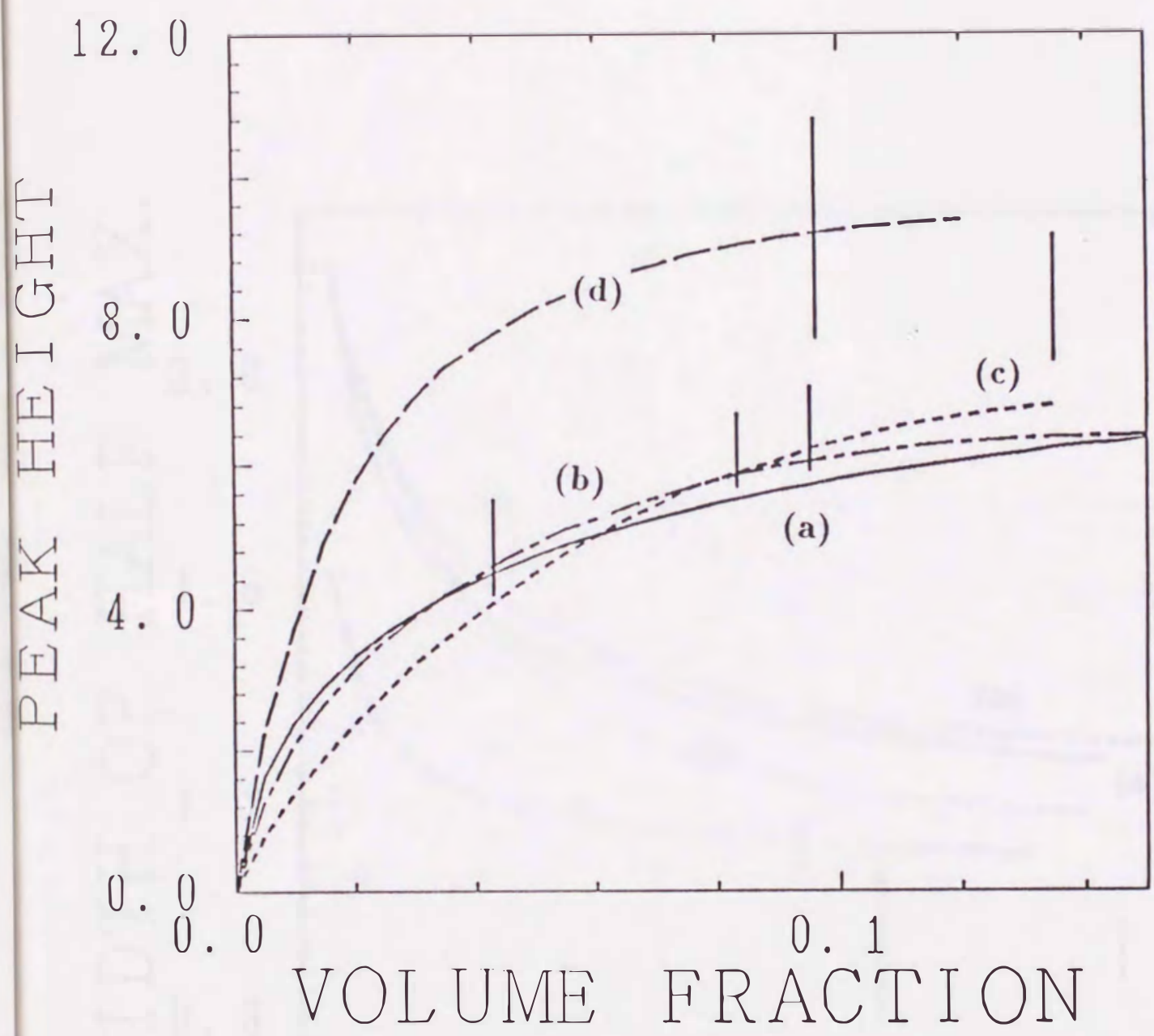

Fig.II-6 


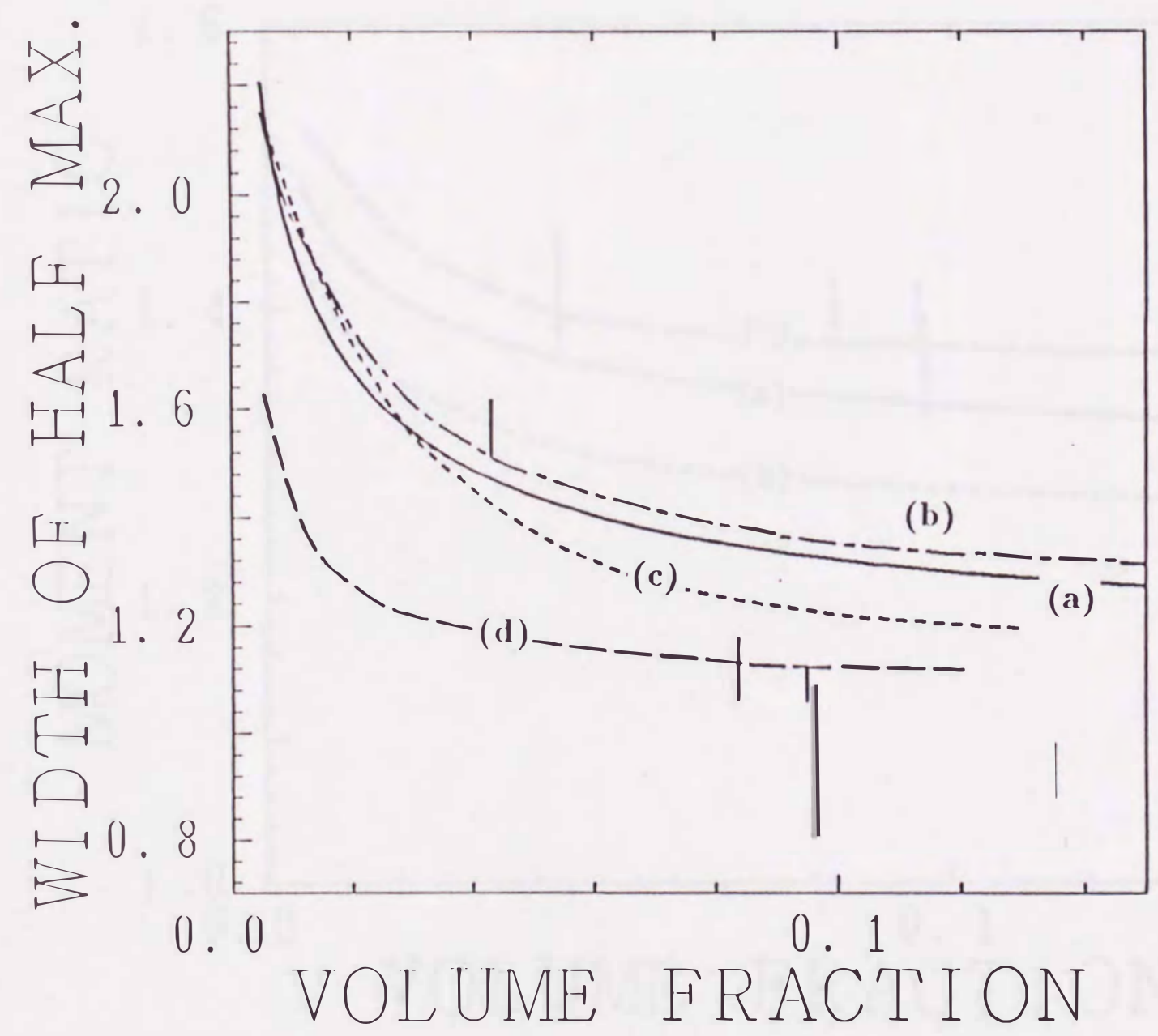

Fig.II-7 


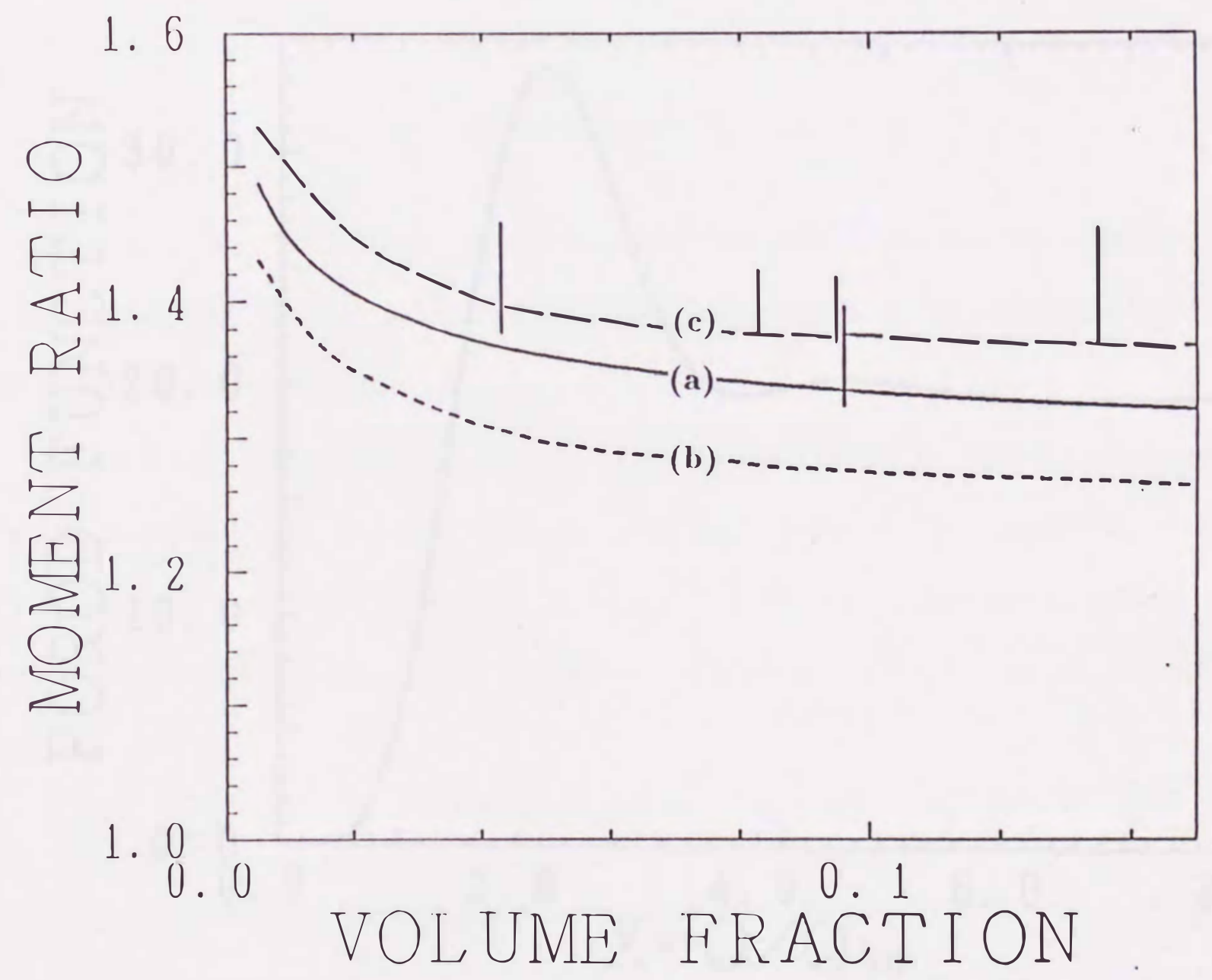

Fig.II-8 


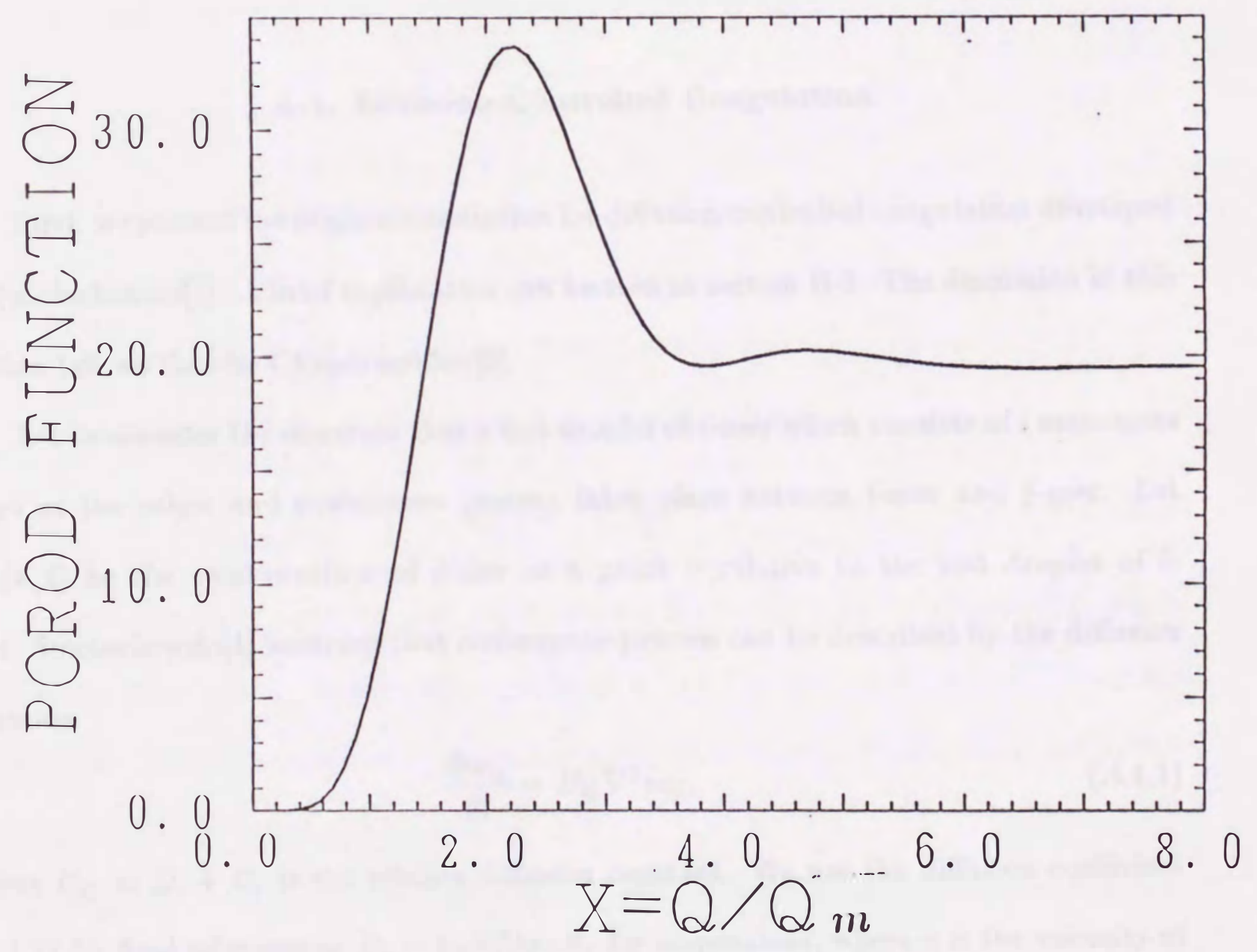

Fig.II-9 


\section{Appendix A: Classical 'Theory of Coagulation Processes}

In $\Lambda$ ppendix $\Lambda$, we review the classical theory in coagulation processes. We obtain rate constants both for diffusion-controlled coagulation and shear-induced coagulation based on the classical picture. We also estimate the effect of forces among droplets within the classical level. Exact solutions of Smoluchowski's rate equation with special rate constants will be discussed in $A$ ppendix $B$.

\section{$\S$ A-1. Diffusion-Controlled Coagulation}

First, we present the original description for diffusion-controlled coagulation developed by Smoluchowski[1]. A brief explanation can be seen in section II-1. The discussion in this section follows that by Chandrasekhar[2].

Let us consider the situation that a test droplet of $i$-mer which consists of $i$ monomers stays at the origin and coalescence process takes place between $i$-mer and $j$-mer. Let $w_{i j}(r, t)$ be the concentration of $j$-mer at a point $\boldsymbol{r}$ relative to the test droplet of $i$ mer. Smoluchowski[1] assuned that coalescence process can be described by the diffusion equation

$$
\frac{\partial w_{i j}}{\partial t}=D_{i j} \nabla^{2} w_{i j}
$$

where $D_{i j}=D_{i}+D_{j}$ is the relative diffusion constant. We use the diffusion coefficient (II.1.T) for fluid mixtures or $D s=k_{B} T / 6 \pi \eta R_{s}$ for suspensions, where $\eta$ is the viscosity of the background fluid. The boundary condition is

$$
w_{i j}(r, t=0)=c_{i} \quad \text { for } \quad r \equiv|r|>R_{i j} \equiv R_{i}+R_{j}
$$




$$
w_{i j}(r, t>0)=0 \quad \text { at } \quad r=R_{i j}
$$

When we assume that the system depends only on the relative distance $r,(\mathrm{~A} .1 .1)$ is reduced to

$$
\frac{\partial\left(r w_{i j}\right)}{\partial t}=D_{i j} \frac{\partial^{2}\left(r w_{i j}\right)}{\partial r^{2}}
$$

The solution of this equation with the condition (A.1.2) is

$$
w_{i j}=c_{i}\left[1-\frac{r}{R_{i j}}+\frac{2 R_{i j}}{\pi^{1 / 2} r} \int_{0}^{\left(r-R_{i j}\right) / 2\left(D_{i j} t\right)^{1 / 2}} \exp \left(-x^{2}\right) d x\right] .
$$

From (A.1.4) the current arriving at the surface $r=R_{i j}$ is given by

$$
J_{i j}=4 \pi D_{i j} R_{i j}\left(r^{2} \frac{\partial w_{i j}}{\partial r}\right)_{r=R_{i j}}=4 \pi D_{i j} R_{i j} c_{j}\left(1+\frac{R_{i j}}{\left(\pi D_{i j} t\right)^{1 / 2}}\right)
$$

Note that there is the correction term to the classical result in (II.1.3) in the above expression, where the term $\xi_{\bullet}=\left(\pi D_{i j} t\right)^{1 / 2}$ can be regarded as a kind of screening length. However in most cases of practical interest the collision interval $\Delta t$ satisfies $\Delta t \gg R_{i j}^{2} / D_{i j}$. With this consideration we obtain the classical coagulation rate

$$
K_{i j}^{c l}=4 \pi D_{i j} R_{i j}
$$

From this type of discussion we can show the existence of the screening length, where the Laplace equation in a steady limit is replaced by the Helmholtz equation. We illustrate an effective medium theory to derive correction to the classical coagulation rate. This discussion is given by the present author.

Let us consider a system with finite density of droplets. The size distribution function obeys the rate equation (I.1.2). The local concentration field $w_{i j}(r, t)$ considered in the above discussion obeys

$$
\frac{\partial w_{i j}}{\partial t}=D_{i j} \nabla^{2} w_{i j}-D_{i j} \xi_{i j}^{-2}+H_{i j}
$$


where

$$
D_{i j} \xi_{i j}^{-2}=\sum_{l=i, j} \sum_{m}^{\infty} K_{l m} w_{l m}
$$

is a sink term for the concentration field and

$$
H_{i j}=\frac{1}{2} \sum_{l=i, j} \sum_{m+n=l} K_{m n} w_{l m} w_{l n}
$$

is a source term. 'The local concentration field is identical to the concentration of clusters a long distance away from a test droplet. The ambiguity of choosing a test droplet appears in the summation of $l$. Under the steady condition $\partial w_{i j} / \partial t=0$, the effective coagulation rate is obtained from the solution of (A.1.7) with a self-consistent condition (A.1.8),

Let us consider a situation similar to that in the derivation of the classical theory. To obtain the steady solution, the source term and the sink term must be balanced as

$$
H_{i j}=D_{i j} \xi_{i j}^{-2} c_{j}
$$

Thus, in the steady state, we have

$$
\left[\nabla^{2}-\xi_{i j}^{2}\right] \delta w_{i j}(r)=0
$$

where $\delta w_{i j}(r) \equiv w_{i j}(r)-c_{j}$. This equation is the Helmholtz equation where $\xi_{i j}$ plays a role of the screening length. The solution of (A.1.11) with the boundary condition of (A.1.2) is given by

$$
w_{i j}(r)=c_{j}\left[1-\frac{R_{i j}}{r} \exp \left[-\frac{r-R_{i j}}{\xi_{i j}}\right]\right]
$$

'Thus, the total current arriving at the reaction surface is given by

$$
J_{i j}=4 \pi D_{i j} R_{i j}\left(r^{2} \frac{\partial w_{i j}}{\partial r}\right)_{r=R_{i j}}=4 \pi D_{i j} R_{i j} c_{j}\left(1+\frac{R_{i j}}{\xi_{i j}}\right) .
$$


Substituting this into $(\Lambda .1 .8)$ we find the screening length $\xi_{i j}$

$$
\xi_{i j}^{-2}=\frac{2 \pi}{D_{i j}} \sum_{l=i, j} \sum_{m} D_{l m} R_{l m} c_{m},
$$

where we take into account the double count of $w_{l m}$ to replace it by $c_{m}$.

'The expressions in (A.1.13) and (A.1.14) are the same as those in (II.1.8) and (II.1.9) or in (II.4.10). 'This surprising result is essentially due to the mean-field treatment in Chapter II.

\section{$\S$ A-2. Shear-Induced Coagulation}

In this section we discuss shear-induced coagulation which is one of most exciting subjects in the field of complex fluids[3-5]. We present the classical theory to obtain the coagulation rate of suspensions in shear flow. The discussion in this section follows that in Ref.[6].

Droplets in uniform shear flow collide with each other due to their relative motion. We assume that droplets moves with straight trajectories. For simplicity, we neglect the elongation of clusters or droplets under the influence of shear flow (velocity $u$ ). 'Therefore, all droplets have spherical forms.

\section{Figure A-1 and Figure A-2}

Look at Fig.A-1 to derive the coagulation rate constant. This figure shows that a droplet with its radius $R_{i}$ interacts with another droplet of radius $R_{j}$ under shear flow. Let $x$ be the vertical axis in Fig. $A-1$. 'The velocity of the vertical component for a droplet at $x$ is given by $x S=x(d u / d x)$ where $S$ is the shear rate. 'Therefore, the current arriving at the shell with the width $d x$ is given by

$$
j_{i j}(\theta) d \theta=c_{j}[x S]\left(R_{i}+R_{j}\right) \sin \theta d x
$$


where the droplet $R_{i}$ exists at the origin in Fig.A-2. In (A.2.1) the terms $[x S]$ and $\left(R_{i}+\right.$ $\left.R_{j}\right) \sin \theta d x$ represent the relative velocity and the cross section in the shell $d x$, respectively. Noting $x=\left(R_{i}+R_{j}\right) \cos \theta$ and $d x=-\left(R_{i}+R_{j}\right) \sin \theta d \theta$ the total flux is given by

$$
J_{i j}=2 \times 2 c_{j} S\left(R_{i}+R_{j}\right)^{3} \int_{0}^{\pi / 2} \sin ^{2} \theta \cos \theta
$$

where $J_{i j} \equiv-4 \int_{0}^{\pi / 2} d \theta j_{i j}(\theta)$. The first coefficent 2 is the freedom of the direction of shear flow, and the second 2 represents two integrals for $\theta$. Therefore we obtain

$$
K_{i j}^{\text {shear }}=\frac{4}{3} S\left(R_{i}+R_{j}\right)^{3}
$$

'This is the classical result of shear-induced coagulation.

It is easy to obtain the growth law of shear-induced coagulation. Using the discussion in section 1-3 the homogeneity of coagulation kernel under shear flow is $\lambda=1$. Therefore, the growth law is

$$
\bar{s}(t) \sim \exp (\text { const.St })
$$

where $\bar{s}(t)$ is the mean droplet size.

\section{$\S$ A-3. The Effect of Force Field to Brownian Coagulation}

If there is a force field among clusters, the form of coagulation rate is changed. The most important force fields are the van der Waals force and the Coulomb force. In this section we discuss the ellect of the Coulomb force field to the rate constant in the Brownian coagulation within the framework of the classical theory. The argument in this section is based on that in R.ef.[6].

Let us consider the case that a droplet with its radius $R_{i}$ stays at the origin and a droplet with radius $R_{j}$ difluses under the effect of the potential field $U_{i j}(r)$, where $r$ is the 
relative distance between $i$ and $j$. 'The flux of $j-$ mer to $i-$ mer can be estimated as

$$
j_{i j}(r)=-D_{i j} \frac{\partial w_{i j}}{\partial r}-\frac{1}{\zeta_{i j}} \frac{\partial U_{i j}(r)}{\partial r} w_{i j}(r)
$$

where $\zeta_{i j}$ is the friction constant and $w_{i j}(r)$ is the concentration of $j-$ mer at $r$. In a steady state the flux passing through reaction radius $r=R_{i j}$ is constant. 'Then we have

$$
J_{i j}=-4 \pi r^{2} j_{i j}(r)=4 \pi r^{2} D_{i j}\left(\frac{\partial w_{i j}}{\partial r}+\frac{w_{i j}}{k_{B} T} \frac{\partial U_{i j}(r)}{\partial r}\right)
$$

where we use $\zeta_{i j}=k_{B} T / D_{i j}$. 'The solution of this equation is given by

$$
w_{i j}(r)=c_{j} \exp \left[-\frac{U_{i j}(r)}{k_{B} T}\right]+\frac{J_{i j} \exp \left[-U_{i j}(r) / k_{B} T\right]}{4 \pi D_{i j}} \int_{\infty}^{r} \frac{\exp \left[U_{i j}(r) / k_{B} T\right]}{x^{2}} d x
$$

where $c_{j}$ is the concentration at $r \rightarrow \infty$. Using the condition for $w_{i j}\left(r=R_{i}+R_{j}\right)=0$ the final expression of current is given by

$$
J_{i j}=\frac{4 \pi c_{j} R_{i j}}{R_{i j} \int_{R_{i j}}^{\infty}\left[\exp \left[U(r) / k_{B} T\right] / x^{2}\right] d x}=\frac{4 \pi D_{i j} R_{i j} c_{j}}{A_{i j}}
$$

Therefore, the coagulation rate has correction in the form of $A_{i j}$.

Now we estimate the effect of the Coulomb force. It seems contrastive the Coulomb field to our model in Chapter II, because the Coulomb force is long ranged, while ours is short ranged. We show that the effect of the Coulomb field is not crucial, at least, for weakly charged systems. 'The potential form in a medium with the dielectric constant $\epsilon$ is

$$
U_{i j}(r)=\frac{z_{i} z_{j} e^{2}}{\epsilon r}
$$

where $e$ is the the elementary electric charge, and $z_{i}$ and $z_{j}$ are charges of $i$ and $j$-mer, respectively. Substituting ( $\Lambda .3 .5)$ into (A.3.4) we obtain

$$
A_{i j}=\frac{1}{y_{i j}}\left(\exp \left(y_{i j}\right)-1\right)
$$


where

$$
y_{i j}=\frac{z_{i} z_{j} e^{2}}{\epsilon k_{B} T R_{i j}}
$$

represents the ratio of the electric potential energy to the thermal energy. In the case of neutral droplets, i.e. $y_{i j}=0$, the correction term is 1 . The correction term is in the region of $0.8 \leq A_{i j} \leq 1.3$ for $-0.5 \leq y_{i j} \leq 0.5[6]$. Therefore, the contribution from the Coulomb force is small, at least, for weakly charged cases. 


\section{Appendix B: Exact Solutions of Smoluchowski's Rate Equation}

In $\Lambda$ ppendix B, we illustrate exact solutions of Smoluchowski's rate equation (I.1.2).

As mentioned in the text, we know only exact solutions for Smoluchowski's rate equation, which are $K_{i j}=1[1], i+j[7]$ and $i j[8]$ in a certain unit. It is possible to solve the equation where the kernel is the linear combinations of exact solved kernels [9]. For simplicity we restrict ourselves to the cases of $K_{i j}=1[1], i+j[7]$ and $i j[8]$.

\section{$\S$ B-1 Exact Solution for $K_{i j}=1$}

First, we discuss the case of size independent kernels[1,2]. This situation can be interpreted as an approximated description of diffusion-controlled coagulation. Substituting

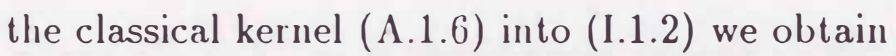

$$
\frac{\partial c_{s}(t)}{\partial t}=4 \pi\left[\frac{1}{2} \sum_{i+j=s} D_{i j} R_{i j} c_{i}(t) c_{j}(t)-\sum_{j=1}^{\infty} D_{s j} R_{s j} c_{s}(t) c_{j}(t)\right] .
$$

Assuming the diffusion constant (II.1.7) the coagulation kernel depends on the following form

$$
K_{i j} \propto\left(i^{1 / 3}+j^{1 / 3}\right)\left(i^{-1 / 3}+j^{-1 / 3}\right) .
$$

$\Lambda$ numerical solution of (B.1.1) with (B.1.2) was given by Friedlander and Wang[10]. In spite of a simplification to derive the above, it is impossible to solve (B.1.1) analytically. Smoluchowski[1] made an additional assumption to solve (B.1.1) as

$$
D_{i} R_{i}=D R=\text { const }
$$

and he also added another assumption

$$
R_{\mathbf{i}}=R_{j}=R
$$


Of course, (B.1.4) is not satisfied in real systems. From (B.1.3) and (B.1.4) we have

$$
D_{i j} R_{i j}=4 D R .
$$

If we introduce the scaled time $\tau=16 \pi D R t$, then (B.1.1) is reduced to the simplified form

$$
\frac{\partial c_{\boldsymbol{s}}(\tau)}{\partial \tau}=\frac{1}{2} \sum_{\boldsymbol{i}+j=\boldsymbol{s}} c_{\boldsymbol{i}}(\tau) c_{j}(\tau)-c_{\boldsymbol{s}}(\tau) M_{0}(\tau)
$$

Thus, the problem is reduced to that with the kernel $K_{i j}=1$. Although such a simplification cannot be justified, it is useful to know analytic properties of Smoluchowski's equation.

It is easy to solve (B.1.6). Let (B.1.6) sum over all sizes $s$ we obtain

$$
\frac{\partial M_{0}}{\partial \tau}=\frac{1}{2} M_{0},
$$

where $M_{0}=\sum_{s} c_{s}$ is the number density of droplets. In this section we use the notation $M_{n}=\sum_{s} s^{n} c_{s}$. The solution of this equation is simply given by

$$
M_{0}(\tau)=\frac{M_{0}(\tau=0)}{1+M_{0}(0) \tau / 2} .
$$

Using (B.1.8) we can successively obtain the solution of $c_{\boldsymbol{s}}$. Considering the equation for $c_{1}$ we have

$$
\frac{\partial c_{1}}{\partial \tau}=-c_{1} M_{1}=-\frac{c_{1} M_{0}(0)}{1+M_{0}(0) \tau / 2},
$$

in other words

$$
c_{1}=\frac{M_{0}(0)}{\left(1+M_{0}(0) \tau / 2\right)^{2}} .
$$

Proceeding in this manner we can prove by induction the following size distribution

$$
c_{s}=M_{0}\left[\frac{\left(M_{0}(0) \tau / 2\right)^{s-1}}{\left(1+M_{0}(0) \tau / 2\right)^{s+1}}\right] .
$$


'Ihis is an exact solution of Smoluchowski's rate equation.

We illustrate a scaling form of (B.1.1). For simplicity we assume the monodisperse initial condition, i,e. $c_{s}(\tau=0)=\delta_{s 1}$ and $M_{0}(0)=M_{1}(\tau)=1$. For large size of clusters and large $\tau$, the solution (B.1.10) has an asymptotic form as

$$
c_{s} \simeq\left(\frac{2}{\tau}\right)^{2} \exp [-x]
$$

where $x=s /(\tau / 2)$. Considering $M_{2} / M_{1}=\tau / 2$ we can interpret $\tau / 2$ as the mean cluster size. 'Thus, (B.1.1) is the standard form of scaling solution (I.3.2) where the scaling function is $F(x)=e^{-x}$.

When we compare (B.1.11) with the numerical result[10], we find the followings: (i) The asymptotic form of numerical scaling function $F(x)$ can be approximately represented by $F(x) \simeq 0.915 e^{-0.95 x}$ for $x \gg 1$, which is also an approximated expression for $x>0.5$. This functional form is similar to $e^{-x}$ in (B.1.11). (ii) The asymptotic form of numerical scaling function for $x \ll 1$ tends to zero. Therefore, the above approximated method cannot be useful for small clusters. In spite of simplification (B.1.4) the analytic method give a qualitative agreement with a numerical method[10].

\section{$\S$ B-2 The Exact Solution of the Rate Equation for $K_{i j}=i j$}

In this section we consider the rate equation for $K_{i j}=i j[8]$. This is a model equation of polymerization.

Polymerization is a typical example of reaction limited aggregation (RLA). Flory[11] estimated the sticking probability of two contacted polymers as $10^{-13}$. Flory assumed that polymers have loopless structures. Let $f$ be the functionality of each monomer. A loopless $s-$ mer has $r_{s}=(f-2) s+2$ reactivity. Assuming the interpenetrativity of two 
contacted polymers, the coagulation rate is proportional to the possible number of reactive sites. 'Therefore, we can use the kernel $K_{i j}=[(f-2) i+2][(f-2) j+2]$ which reduces to $K_{i j} \sim f^{2} i j$ for large $f$. In the last decade, we find that the solution of the rate equation with this kernel displays a gelation transition[8].

We illustrate the gelation as a solution of the rate equation. Although Spouge[9] analytically solved the rate equation with $K_{i j}=[(f-2) i+2][(f-2) j+2]$ assuming the monodisperse initial condition, it would lead to needless complications. Therefore, we restrict ourselves to a simple case $K_{i j}=i j$ in this section. Let us consider the following

$$
\frac{\partial c_{s}(t)}{\partial t}=\frac{1}{2} \sum_{i+j=s} i j c_{i}(t) c_{j}(t)-c_{s}(t) M_{1}(t)
$$

with the choice of a suitable unit. It is easy to demonstrate the gelation using (B.2.1) Multiplying $s^{2}$ to (B.2.1) and summing over $s$ we obtain

$$
\frac{\partial M_{2}}{\partial t}=M_{2}^{2}
$$

The solution is simply given by

$$
M_{2}(t)=\frac{M_{2}(0)}{1-M_{2}(0) t}
$$

where $M_{2}(t)$ diverges at $t=1 / M_{2}(0)$. We can interpret this singularity as an emergence of an infinite cluster

'The size distribution of (B.2.1) can be obtained. For simplicity, we assume the monodisperse initial condition, i.e. $c_{s}(t=0)=\delta_{s 1}$. We introduce the generating functions as

$$
g(x, t) \equiv \sum_{s} c_{s}(t) \exp (-s x) \quad \text { and } \quad f(x, t) \equiv \sum_{s} s c_{s}(t) \exp (-s x)
$$


We denote the initial values of generating functions as $u(x)=f(x, 0)=-e^{-x}$ and $v(x)=$ $g(x, 0)=e^{-x}$. Multiplying (B.2.1) with $s e^{-s x}$ and summing over $s$ we obtain a partial differential equation for $f(x, t)$ as

$$
\frac{\partial f(x, t)}{\partial t}=\frac{\partial f(x, t)}{\partial x}[1-f(x, t)]
$$

where we use $f(0, t)=1$. Introducing the inverse function $x=X(f, t),($ B.2.5) can be reduced to

$$
\frac{\partial X(f, t)}{\partial t}=1-f(x, t)
$$

where we use $\partial f / \partial x=1 /(\partial X / \partial f)$ and $(\partial f / \partial t)=-(\partial X / \partial t) /(\partial X / \partial f)$. The solution of (B.2.6) with the initial condition $f(x, 0)=u(x)$ is

$$
x=u^{-1}(f)-t f+t
$$

where $u^{-1}(f)=x$ is the inverse function of $f=u(x)$. For later conveniences we adopt the parametric form as

$$
x=s-t u(s)+t ; \quad f(x, t)=u(s)
$$

Once the generating function $f(x, t)$ is obtained, the size distribution can be found by the expansion of the exponential part. We introduce $F(z, t)$ and $U(z)$ as

$$
F^{\prime}(z, t)=f(x, t)=\sum_{s=1}^{\infty} s z^{s} c_{s}(t) \quad \text { and } \quad U(z)=u(x)=\sum_{s=1}^{\infty} s z^{s} c_{s}(0)
$$

Using the notations in (B.2.9), (B.2.8) can be rewritten as

$$
z=y \exp [-t U(s)+t] ; \quad F=U(y)
$$

where $y=e^{-s}$. We introduce Lagrange's expansion[12] of $F(z, t)$ in powers of $z-z_{0}$ as

$$
F(z, t)=U(y)+\sum_{s=1}^{\infty} \frac{\left(z-z_{0}\right)^{s}}{s !}\left[\left(\frac{d}{d y}\right)^{s-1} U^{\prime}(y)\left(\frac{\left.y-y_{0}\right)}{z(y)-z_{0}}\right)^{s}\right]_{y=y_{0}} .
$$


To obtain the size distribution we expand $F(z, t)$ about $z_{0}=0$ and $y_{0}=U\left(y_{0}=0\right)=0$ 'Thus, we obtain

$$
c_{s}(t)=\left(t k^{2} k !\right)^{-1} \exp (-k t)\left[\left(\frac{d}{d y}\right)^{s} \exp \{s t U(y)\}\right]_{y=0}
$$

Using $u(x)=-e^{-x}$ and $U(z)=-z$ we obtain the final expression of $c_{\mathrm{s}}$ as

$$
c_{s}(t)=t^{s-1} s^{s-2} \exp (-s t) / s !
$$

where the solution is valid only for $t \leq 1$. In the limit of $t \rightarrow 1$ the asymptotic solution for $s \gg 1$ is given by

$$
c_{s}(t)=(2 \pi)^{-1 / 2} s^{-5 / 2}
$$

where we use the Stirling formula.

'The solution of (B.2.13) and (B.2.14) describe gelation at $t=1$. In fact, there is a net mass flux to an infinite cluster. 'The mass flux is defined by

$$
J(t)=\lim _{L \rightarrow \infty} \sum_{s=1}^{L} s \frac{\partial c_{s}(t)}{\partial t} .
$$

It is clear that $J(t)=0$ in the usual state. When there is a negative current, the missing mass is absorbed in an infinite cluster. That is nothing but gelation. Substituting (B.2.13) into (B.2.15) we find

$$
J(t)=-1
$$

Then the gelation occurs at $t=1$.

In physical cases, polymers are not interpenetrative. Therefore, the above discussion is only an approximated description of polymerization.

Appendix B-3: Exact Solution for $K_{i j}=i+j$ 
'The final exactl $y$ solved model is the rate equation with $K_{i j}=i+j$ [7]. This problem can be transformed into that of $K_{i j}=i j[13]$.

'The rate equation with $K_{i j}=i+j$ can be written as

$$
\frac{\partial c_{s}(t)}{\partial t}=\sum_{i+j=s} i c_{i}(t) c_{j}(t)-s c_{s}(t) M_{0}(t)-c_{s}(t)
$$

Introducing new variables

$$
\tau=\int_{0}^{t} d t^{\prime} M_{0}\left(t^{\prime}\right) \text { and } n_{s}(\tau)=c_{s}(t) / M_{0}(t)
$$

the rate equation (B.3.1) is reduced to

$$
\frac{\partial n_{s}(\tau)}{\partial \tau}=s^{-1} \sum_{i+j=s} i(i j) n_{i}(\tau) n_{j}(\tau)-s n_{s}(\tau)=\frac{1}{2} \sum_{i+j=s}(i j) n_{i}(\tau) n_{j}(\tau)-s n_{s}(\tau) \sum_{j=1}^{\infty} j n_{j}(\tau)
$$

where we use $\partial M_{0} / \partial t=-M_{0}$. Equation (B.3.3) is identical to (B.2.1). For the monodisperse initial condition, $\tau$ is given by $\tau=1-e^{-t}$. Therefore, the critical time $\tau=1$ as in Appendix B-2 becomes $t \rightarrow \infty$. Since $n_{s}$ is identical to (B.2.13), the explicit form of size distribution is

$$
c_{s}(\tau)=(1-\tau)(s \tau)^{s-1} \exp [-s \tau] / s !
$$

This solution was directly obtained by Scott[7]. 


\section{References}

[1]. M.von Smoluchowski, Physik Z.17 (1916) 585.

M.von Smoluchowski, Z.Phys.Chem.92 (1918) 129.

[2]. S.Chandrasekhar, Rev.Mod.Phys. 15 (1943) 1.

[3]. A.Onuki, Phys.Rev.Lett.62 (1989) 2472; J.Phys.Soc.Jpn.59 (1990) 3427.

[4]. E.Ilelfand and G.H. Fredrickson, Phys.Rev.Lett.62 (1989) 2468.

[5]. 'T.Ohta, II.Nozaki and M.Doi, J.Chem.Phys.93 (1990) 2664.

M.Doi and 'T.Ohta, J.Chem.Phys. in press.

[6]. S.K.Friedlander, Smoke, Dust and Haze(Wiley, New York 1977).

[7]. W.I.Scott, J.Atmos.Sci.25(1968) 54.

[8]. R.M.Ziff, M.II.Ernst and E.M.Hendriks, J.Phys.A16 (1983) 2293.

[9]. J.L.Spouge, J.Phys.A 16(1983) 767.

[10]. S.K.Friedlander and C.S.Wang, J.Colloid Interface Sci. 22 (1966) 126.

[11]. P.J.Flory, Principles of Polymer Chemistry (Cornell Univ.Press, New York 1953).

[12]. M.Abramowitz and I.Stegun, Handbook of Mathematical Functions (Doover, New York 1974).

[13]. E.M.IIendriks, R.M.Ziff and M.II.Ernst, J.Stat.Phys. 31 (1983) 519. 


\section{Figure Captions}

Fig. $\Lambda$ 1: $\Lambda \mathrm{n}$ illustration of a collision of droplets under shear flow. The lower droplet movers faster than the upper one. 'Then they collide each other.

ling. $\Lambda 2: \Lambda$ Geometric realtion of shear-induced coagulation. 'The flow is perpendicular to the paper. 'The center of droplet $R_{\mathbf{i}}$ is at the origin. 


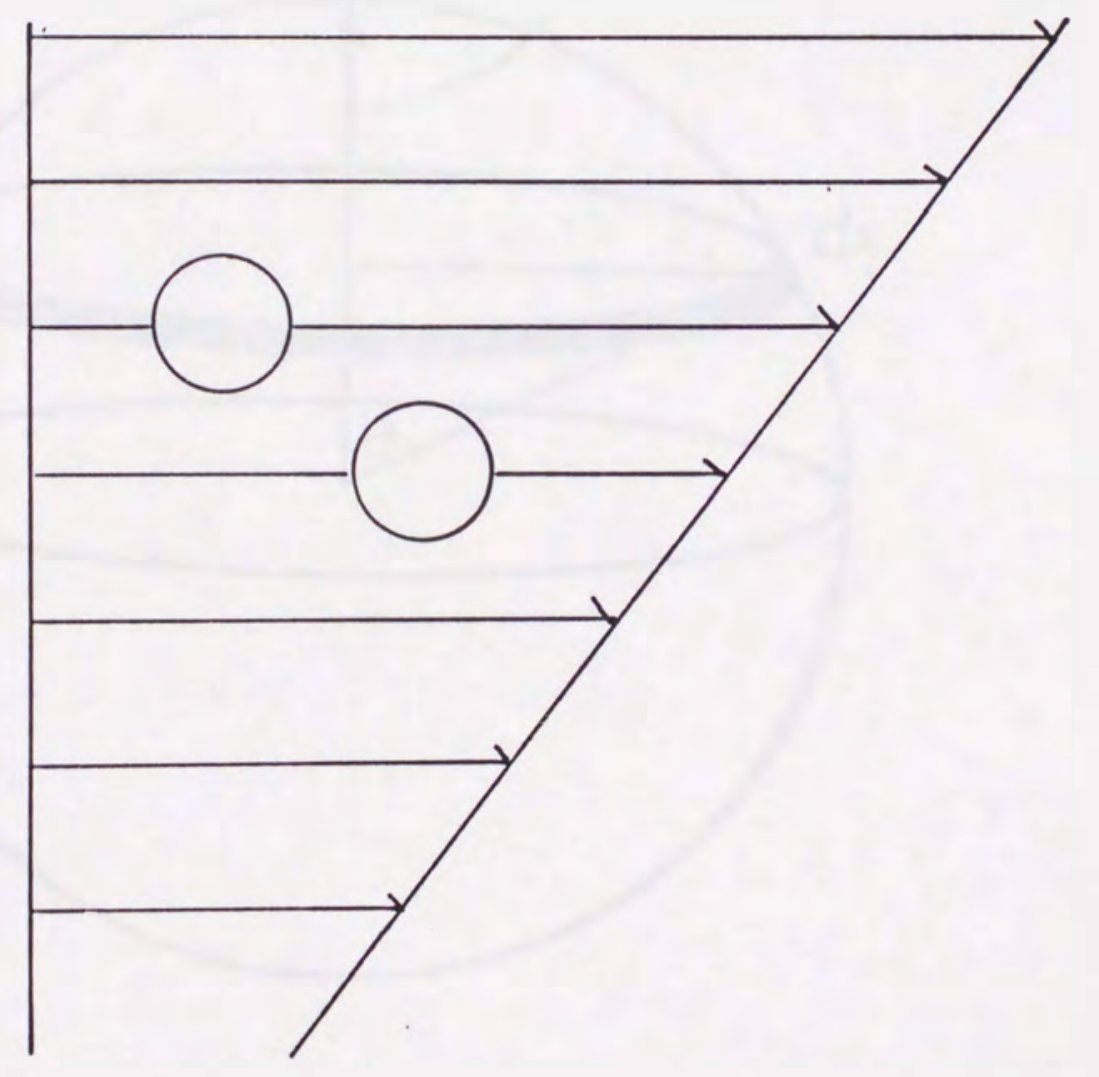

Fig.A-1 


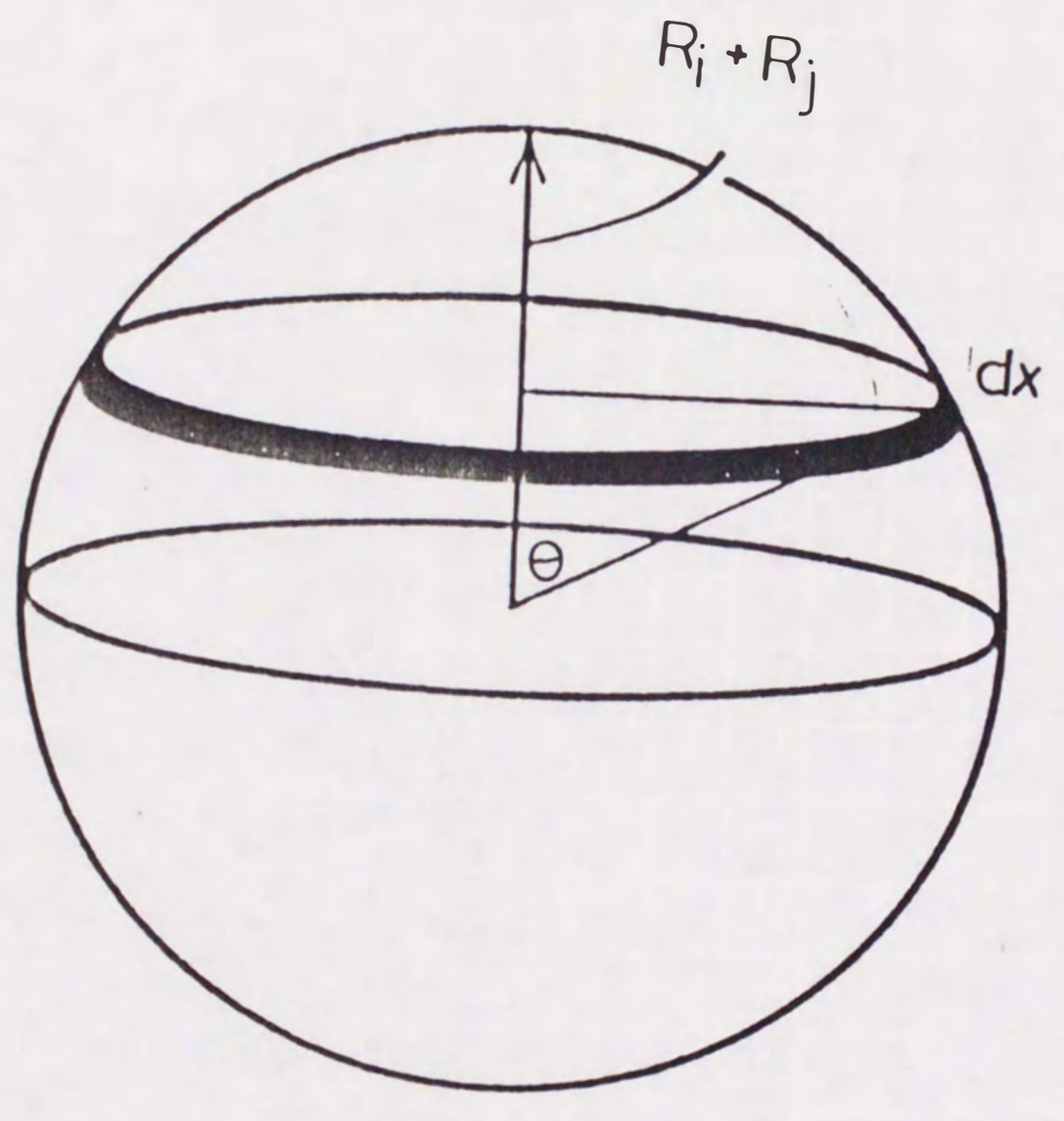

Fig.A-2 


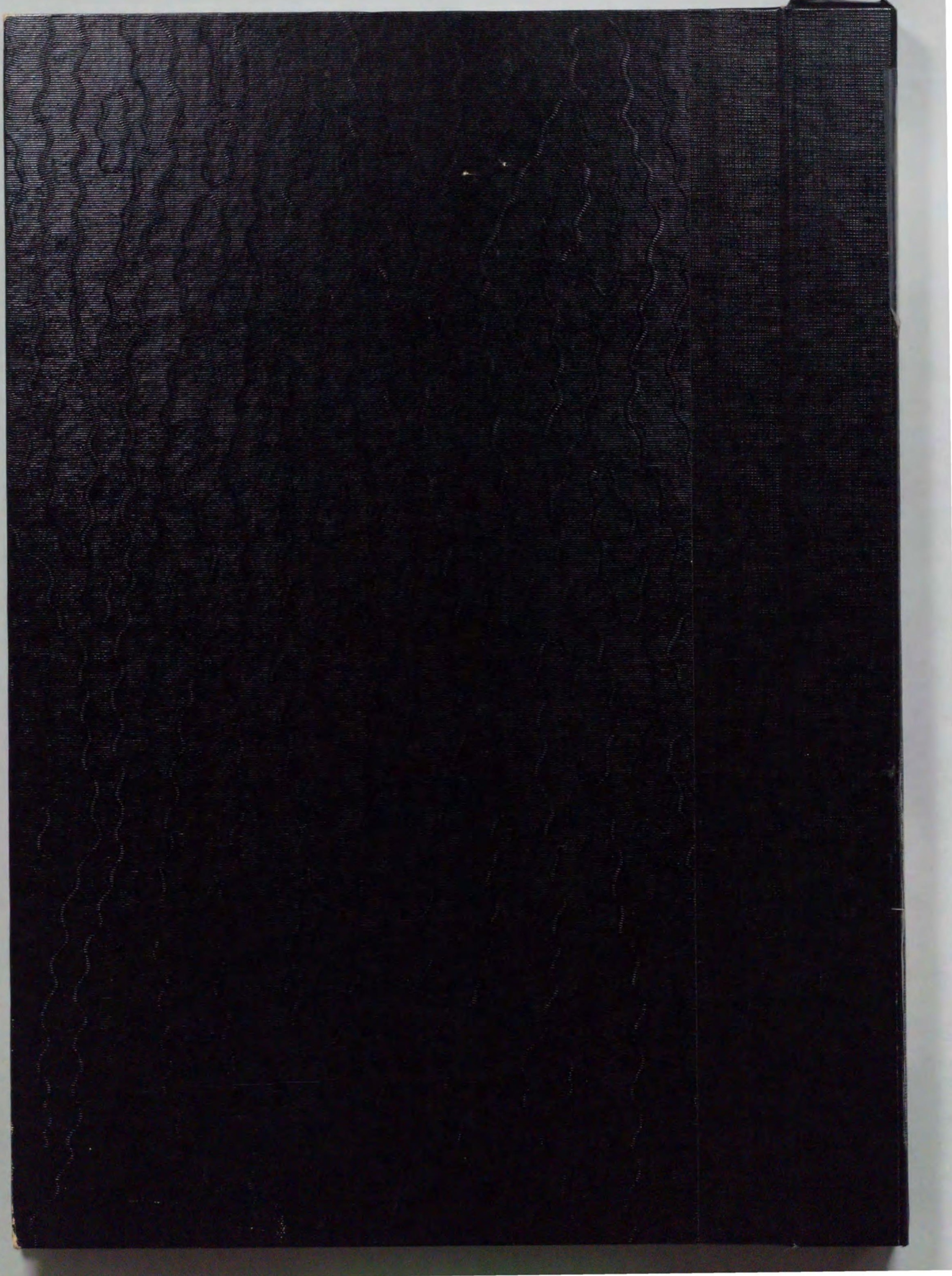




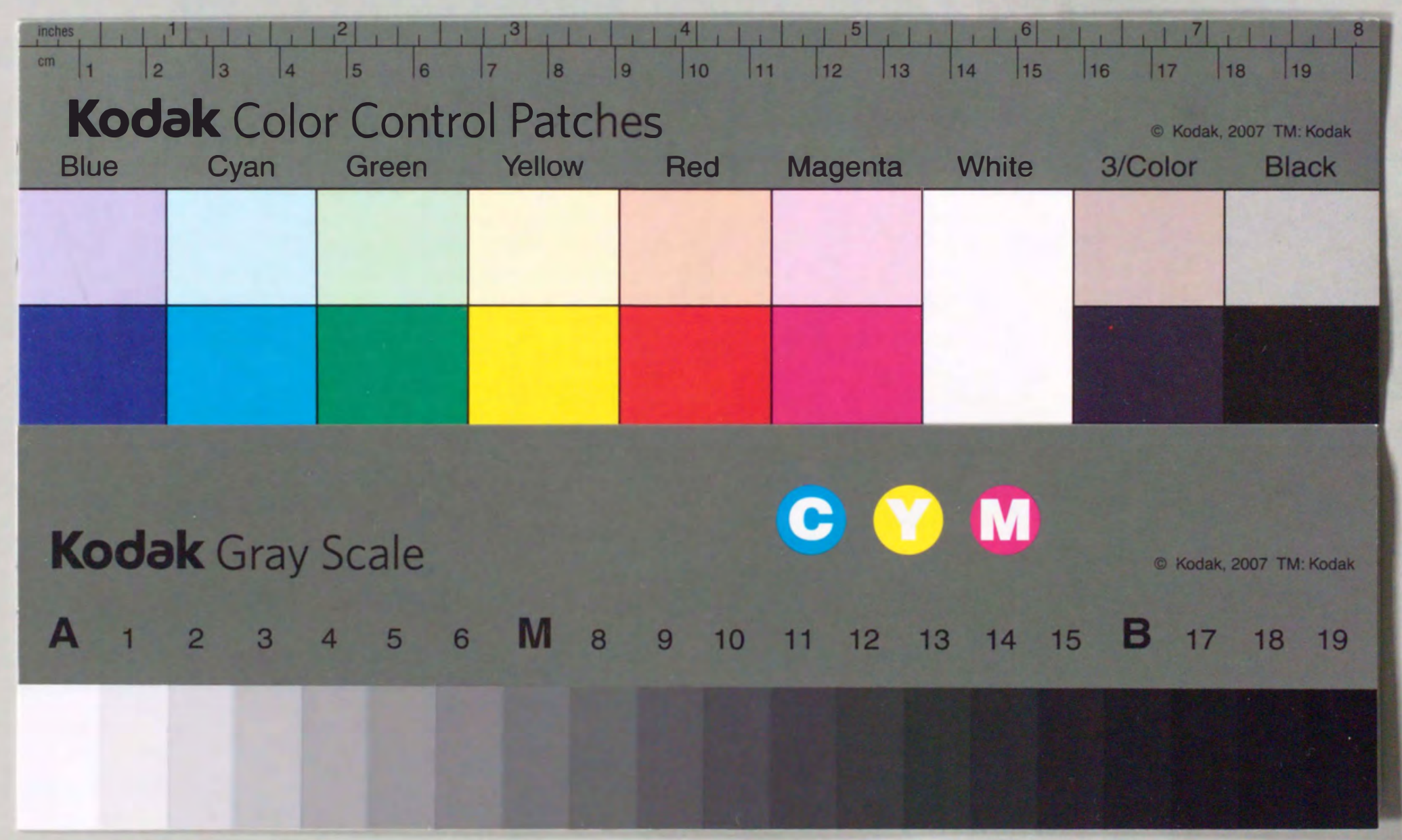

